\title{
„Der Herr ist mein Hirte“ - Konzeptuelle Metapher als Lebenswirklichkeit
}

\author{
JAKOB BÖCKLE (UNIVERSITY OF SOUTH AFRICA AND THE UNIVERSITY \\ OF VIENNA)
}

\begin{abstract}
By means of metaphor, the less tangible can be made more tangible. This also applies to the declaration "The LORD is my shepherd" (Ps 23:1b). For a detailed approach to this declaration and the psalms, the focus here is first on conceptual perception (of God) and the Conceptual Metaphor Theory. It is then applied to the declaration, "The LORD is my shepherd," unfolded in its radiant effect on the entire psalm and merged again into one. The aim is to prove that metaphorical conceptualisation makes the world shine in a new light and become experienceable, as the psalmist experienced in the encounter with God, making himself perceptible to him. Concretely, the psalmist explores the world of the Ancient Near East and of the confidential personal relationship between himself and God in the image of the shepherd-king and the human being-sheep. He underlines the covenant relationship between the Lord and the vassal - and thus the possibility of becoming a reality in one's personal life.
\end{abstract}

KEYWORDS: Psalm 23, (absolute) metaphor, reality of life, conceptualisation, YHWH, my shepherd - sheep (and human being), suzerain - vassal

\section{A METAPHERTHEORIE ALS GRUNDLAGE}

\section{$1 \quad$ Metapher - Konzeptualisierung - Embodied Cognition - Mentale Simulation}

Die Metapher, als Königsweg rhetorischer Vermittlung und Durchdringung des (sprachlichen) Alltags, spielt in der Bibel eine zentrale Rolle. ${ }^{1}$ Ihre vielfältige

* Submitted: 01/12/2020; peer-reviewed: 20/04/2021; accepted: 05/05/2021. Jakob Böckle, "'Der Herr ist mein Hirte': Konzeptuelle Metapher als Lebenswirklichkeit," Old Testament Essays 34 no. 1 (2021): 189 - 217. DOI: https://doi.org/10.17159/23123621/2021/v34n1a11.

1 Für das Thema Metapher im Alten Testament im Allgemeinen und in den Psalmen im Besonderen vergleiche exemplarisch, Pierre J. P. van Hecke, Metaphor in the Hebrew Bible (Leuven: Peeters, 2005); Ders. und Antje Labahn, Metaphors in the 
Anwendung ist in der Sprache der Bibel und im Besonderen in der poetischen Literatur des Alten Testaments und damit in der wohl prominentesten Aussage eines Psalmisten (als Fokusstelle für den vorliegenden Aufsatz), „JHWH ist mein Hirte“" (Ps 23,1b), nicht allein Teil der Konzeptualisierung im Allgemeinen, sondern desgleichen Teil der Konzeptualisierung Gottes im Besonderen. Der Begriff der Konzeptualisierung entstammt der Kognitiven Linguistik und meint Wahrnehmung in Konzepten oder konzeptuelle Wahrnehmung. Das heißt, die Metapher dient in der Bibel vielfach als Werkzeug zur, dem Leser nahebringenden Wahrnehmung im Allgemeinen und der Wahrnehmung Gottes im Besonderen - und das in der aktivierenden Unterlegung von Konzepten. ${ }^{2}$

Die Zahl der Untersuchungen der Bibel in Anwendung der Kognitiven Linguistik ist mittlerweile groß. ${ }^{3}$,Im Gegensatz zu vielen anderen Ansätzen in der Linguistik ist die kognitive Linguistik weniger ein gebrauchsfertiges Paradigma, sondern eher eine spezielle Auffassung von und ein besonderer Zugang zur Sprache.“ Entsprechend könnte metaphorisch konzeptuell gesagt werden, , kognitive Linguistik ist ein Archipel “. ${ }^{4}$ Ein solches Archipel-Konzept „,besteht aus verschiedenen Theorie-Inseln“, denen unterschiedliche Forschungsgebiete (wie die Psycho-, Neuro- und Computerlinguistik) zugrunde liegen, aber die ,trotzdem bestimmte Vorstellungen und Grundannahmen [...] gemeinsam haben“. Dabei erweist sich die wohl wichtigste (gemeinsame) Grundannahme ,in dem Versuch, linguistische Phänomene - wie die Grammatik - nicht nur im Hinblick auf die Sprache, sondern auf allgemeine kognitive, perzeptive und körperliche Fähigkeiten zu erklären". 5

Psalms (Leuven: Peeters, 2010); Danilo Verde und Antje Labahn, Networks of Metaphors in the Hebrew Bible (Leuven: Peeters, 2020).

2 Der vorliegende Aufsatz entspringt Untersuchungen für meine an der University of South Africa (UNISA) angenommene Doktorthesis „Die Reden Gottes an Ijob (Ijob 38,1-42,6) in ästhetisch theologischer Perspektive“. In der Thesis ist zwar der Begriff der konzeptuellen Wahrnehmung zentral, das Thema Metapher wird aber nicht vertiefend entwickelt. Genau dort knüpft der vorliegende Aufsatz an.

3 Allein Johan de Joode, Metaphorical Landscapes and the Theology of the Book of Job: An Analysis of Job's Spatial Metaphors (Leiden: Brill, 2018), 12-13 nennt 23 Arbeiten als "a few examples" für viele, die in den "last two decades" Teile und Bücher der Bibel dahingehend untersuchen.

4 Für zitierten Satz und Satzteil Moiken Jessen, Johan Blomberg und Jörg Roche, Kognitive Linguistik (Tübingen: Narr Francke Attempto, 2018), 17.

5 Für die zitierten Satzteile Jessen, Blomberg und Roche, Kognitive Linguistik, 17; für das Thema des Absatzes (außer dem ersten Satz) ausführlicher Gert Rickheit, Sabine Weiss und Hans-Jürgen Eikmeyer, Kognitive Linguistik: Theorien, Modelle, Methoden (Tübingen: A. Francke, 2010), 9-14, 289-294. 
Innerhalb der Kognitiven Linguistik bilden Konzepte grundlegende Bausteine der Wahrnehmung beziehungsweise der Kognition (siehe im Folgenden) ab. Entsprechend können Konzepte als diskret unterscheidbare mentale Repäsentationen, als Informations- und Organisationseinheiten des Denkens gefasst werden. Im Zuge der im Gehirn beständig einströmenden Informationen in unzählbar vielfältigstem $\mathrm{Ma} \beta$ ermöglichen Konzepte kategoriell geordnete Weltwahrnehmung, Welterkenntnis und Weltinterpretation - was Konzepte als Kategorienkonzepte fassbar macht. Sie ermöglichen Weltwissen und Sprachwissen und gründen zugleich auf diesen. Sie ermöglichen Weltverstehen in Bildern, Begriffen, Kategorien - und darüber hinaus. Auf ihnen gründend geschieht Konzeptualisierung, konzeptuelle Wahrnehmung. Des Weiteren spricht für Kövecses ${ }^{6}$ ein Konzept beziehungsweise ein konzeptuelles Feld von jeglicher koherent oder ganzheitlich strukturierten Erfahrung.

Hier herein würden in aktueller (aber nicht unumstrittener) Forschungsgewichtung der Kognitiven Linguistik Aspekte der embodied cognition spielen. Denn die Modelle der embodied cognition verfolgen die These, dass insofern „eine Verbindung von Sinneswahrnehmung und inneren mentalen Zuständen kognitiver Prozesse“ besteht, als dass „Erfahrungen in Form von sensor[isch]-motor[ischen] Interaktionen des Körpers mit seiner Umgebung [...] elementare Voraussetzung für jegliche neuronale Repräsentation der Umwelt im Gehirn“ sein können. ${ }^{7}$ Das heißt, tatsächliche Handlungen sind neuronal im Gehirn wiedergespiegelt und werden dort auch dann aktiviert (beziehungsweise simuliert), wenn betreffende Handlungen und Ereignisse nicht tatsächlich ausgeführt, sondern lediglich in Form von Begriffen oder Bildern gedacht, oder auch wenn die Handlungen und Ereignisse lediglich beobachtet werden. ${ }^{8}$

\section{Konzeptualisierungstheorie der Metapher - Blumenbergs ,absolute Metapher“"}

Wenn also mit Blick auf das Alte Testament und hier im Besonderen mit Blick auf die zu Grunde legende Aussage von Ps 23 (,Der Herr ist mein Hirte“) die

6 Zoltán Kövecses, Metaphor: A Practical Introduction (Second Edition; Oxford: University Press, [2002] 2010), 4; vergleiche Eckard Rolf, Metaphertheorien: Typologie, Darstellung, Bibliographie (Berlin: Walter de Gruyter, 2005), 236.

7 Horst M. Müller, "Embodied Cognition, Embodiment," in Metzler Lexikon Sprache (Hg. Helmut Glück und Michael Rödel; 5., aktualisierte und überarbeitete Auflage; Stuttgart: J. B. Metzler, 2016), 174.

8 Hierzu umfassend Rickheit, Weiss und Eikmeyer, Linguistik; Elisabeth Wehling, Politisches Framing: Wie eine Nation sich ihr Denken einredet - und daraus Politik macht (Köln: Herbert von Halem Verlag, 2016). 
Anwendung von Metapher und Konzeptualisierung im Sinne von konzeptueller Wahrnehmung, von mentaler Simulation, von ganzheitlich organisierter Erfahrung des Dargestellten, untersucht werden will, dann liegt die Anwendung der Konzeptualisierungstheorie der Metapher (original Conceptual Metaphor Theory) nach Lakoff und Johnson, ${ }^{9}$ die mittlerweile von Lakoff und weiteren Autoren aktualisierend fortgeführt wird, ${ }^{10}$ am nächsten. Da einige Autoren die Konzeptualisierungstheorie der Metapher als kognitive Theorie und damit als der Kognitiven Linguistik entspringend ansehen, ${ }^{11}$ wird einmal mehr deutlich, dass innerhalb der Konzeptualisierungstheorie die (konzeptuelle) Wahrnehmung zentral ist. Denn bereits der Begriff der Kognition spricht von jeglichem Geschehen bewusster Aisthesis und Noesis, von (sensorischer) Wahrnehmung und (mentaler) Wertnehmung (also dem geistigen, einsichtsvollen Erfassen, Beurteilen, Bewerten und Kategorisieren des Wahrgenommenen). ${ }^{12}$

Innerhalb der überbordenden Fachliteratur zur Metapher ist man allerdings regelmäßig darum bedacht, Lakoffs und Johnsons Metaphertheorie als zwar schillernd und unabkömmlich, aber doch als simplifizierend mit

9 George Lakoff und Mark Johnson, Leben in Metaphern: Konstruktion und Gebrauch von Sprachbildern (Neunte Auflage; Heidelberg: Carl-Auer, [1980, 1997] 2018).

10 Exemplarisch durch George Lakoff und Mark Johnson, Philosophy in the Flesh: The Embodied Mind and Its Challenge to Western Thought (New York: Basic Books, 1999); Kövecses, Metaphor; Ders., Extended Conceptual Metaphor Theory (New York: Cambridge University Press, 2020); George Lakoff und Elisabeth Wehling, Auf leisen Sohlen ins Gehirn: Politische Sprache und ihre heimliche Macht (Heidelberg: Carl-Auer, [2008] 2016); Wehling, Framing.

11 Referiert von Rolf, Metaphertheorien, 235; Matthias Junge, Metaphern und Gesellschaft: Die Bedeutung der Orientierung durch Metaphern (Wiesbaden: Verlag für Sozialwissenschaft, 2011), 8.

12 Für Philip G. Zimbardo, Psychologie (6. neu bearbeitete und erweiterte Auflage; Berlin: Springer, 1995), 357 bezeichnet Kognition ,alle Formen des Erkennens und Wissens“. H. Wagenknecht, „Kognition“, in Lexikon der Psychologie (Wilhelm Arnold, Hans Jürgen Eysenck und Richard Meili; Augsburg: Bechtermünz Verlag, [1971] 1996), 1085 definiert Kognition als „Ausdruck für jeden Proze $\beta^{“,}$, durch den bewusste „Kenntnis von einem Objekt" möglich wird. „Wahrnehmung, Erkennen, Vorstellen, Urteilen [...] " sind Teil der Kognition. Bereits griechisch $\gamma \mathrm{\iota}(\gamma) v \omega \dot{\sigma \kappa} \omega \omega$ und lateinisch cognosco, wovon Kognition abgeleitet ist, sprechen von „erkennen; wahrnehmen; wissen; urteilen."Wilhelm Gemoll, Griechisch-deutsches Schul- und Handwörterbuch (Neunte Auflage; Durchgesehen und erweitert von Karl Vretska mit einer Einführung in die Sprachgeschichte von Heinz Kronasser; München: Freytag Verlag, [1954] 1988), 173; Langenscheidts Großes Schulwörterbuch Lateinisch-Deutsch (Bearbeitet von Dr. Erich Pertsch, auf Grundlage des Menge-Güthling. Erweiterte Neuausgabe. Berlin: Langenscheidt, 1983), 209-210. 
(unterstellt) übermäßigem Eigenanspruch einzuordnen. ${ }^{13}$ Der vorliegende Aufsatz sieht aber doch Lakoffs und Johnsons Ansatz des Metapherverstehens (und dessen aktualisierende Fortführungen) als geeignetes Werkzeug für das Verstehen eines zentralen Aspekts der (mindestens) poetischen Literatur des Alten Testaments an. ${ }^{14}$

Dennoch sollen für den vorliegenden Aufsatz innerhalb mittlerweile beinah ungezählter Metaphertheorien ${ }^{15}$ Aspekte lediglich eines weiteren Interpretationsmodells der Metapher einbezogen werden. Wohl der große, sich noch auf Aristoteles gründende „Metaphorologe“ und Philosoph ist Hans Blumenberg. ${ }^{16}$ Blumenberg versteht Metapher zunächst als „Vorfeld des Begriffs“" als „Vorgriff“, während eine absolute Metapher nicht allein Vorfeld und Vorgriff ist, sondern ,fundierender ,Untergrund“ oder umgreifender ,Horizont“" jeglicher ,Begrifflichkeit““ ${ }^{17}$

Für die absolute Metapher, von Kant als Symbol bezeichnet, ist es nicht möglich, hinter sie in eigentlicher Begrifflichkeit zurückzugehen. Sie besetzt eine durch sonstige Begriffe nicht füllbare Leerstelle. ${ }^{18}$ Sie lässt sich ,nicht ins Eigentliche, nicht in die Logizität zurückholen“" ${ }^{19}$ Vielmehr bringt sie selbst das

13 So exemplarisch Anselm Haverkamp, Metapher - Mythos - Halbzeug: Metaphorologie nach Blumenberg (Berlin: Walter de Gruyter, 2018), 2; Ralph Müller, Die Metapher: Kognition, Korpusstilistik und Kreativität (Paderborn: Mentis, 2012), 29; Rolf, Metaphertheorien, 235-241; zum Teil referiert von Jakob Böckle, Ijob 28 in ästhetisch-theologischer Perspektive: Wahrnehmung Gottes und der Weisheit als Herausforderung des Lebens (Wien: LIT, 2018), 85.

14 Für die Anwendung der Konzeptualisierungstheorie der Metapher mit Blick auf die Bibel vergleiche Lance R. Hawley, Metaphor Competition in the Book of Job (Göttingen: Vandenhoeck \& Ruprecht, 2018); De Joode, Landscapes; Böckle, Ijob 28. De Joode, Landscapes, 12-13 nennt weitere Arbeiten.

15 Hierzu Rolf, Metaphertheorien; auch Haverkamp, Metapher; Böckle, Ijob 28, 8384.

16 So einleitend wiederum Haverkamp, Metapher, 2.

17 Referiert von Dirk Mende, „Vorwort. Begriffsgeschichte, Metaphorologie, Unbegrifflichkeit", in Metaphorologie: Zur Praxis von Theorie (Hg. Anselm Haverkamp und Dirk Mende; Frankfurt am Main: Suhrkamp, 2009), 9, vergleiche 7-9; vergleiche auch die „Kernaussage 32“ bei Rolf, Metaphertheorien, 275-276.

18 Hans Blumenberg, Paradigmen zu einer Metaphorologie (Frankfurt am Main: Suhrkamp, [1960] 2013), 173.

19 Blumenberg, Paradigmen, 14. So ist etwa das Sein in seiner Gesamtheit für den Menschen nicht fassbar. Desgleichen ist es nicht möglich, an Stelle des Begriffes Sein den „eigentlichen“, das Sein als Ganzes und im Einzelnen fassenden Begriff einzusetzen. Es ist nicht möglich, hinter den metaphorischen Begriff des Seins in der Anwendung der eigentlichen Begrifflichkeit zurückzutreten, darum absolute Metapher. 
Eigentliche zum Ausdruck, ist die Erschließung einer neuen Welt, ist tertium comparationis. Die absolute Metapher verhilft - mit Kant gesprochen - dem reinen Verstandesbegriff (der Idee), der als solcher der sinnlichen Wahrnehmung (Anschauung) nicht angemessen zugänglich ist, zur denkbaren „Versinnlichung“. So ist die Schönheit Symbol für die Sittlichkeit, denn jene unterweist den Gewahrenden, über diese so wie über jene zu denken. ${ }^{20}$

Blumenberg zählt zu den absoluten Metaphern Begriffe wie Welt, Geschichte, Mensch, Sein, Leben, Freiheit und auch Gott. Sie sind - so Blumenberg - Indiz des Denkens über sie in einer gewissen Epoche. ${ }^{21}$

\section{Metapher als Wirklichkeitserschließung und Handlungswirkung}

Metapher, vor allem bewusst eingesetzt, ist (auch) uneigentliche Rede, ist Rede in nicht wörtlicher Sprachverwendung. Der Produzent verwendet geschrieben oder gesprochen Begriffe in nicht wörtlicher Bedeutung beziehungsweise er überträgt Begriffe in einen bedeutungsmäßig unüblichen Kontext (produktionsästhetischer Aspekt), die der Rezipient im Gesamt des Geschriebenen oder Gesprochenen einschließlich von dessen Kontext in ihrer Uneigentlichkeit oder kontextuellen Unüblichkeit zu erkennen und in der einen neuen Gesamtheit der Aussage zu dekodieren herausgefordert ist. Hierbei besteht Raum persönlicher Interpretation (rezeptionsästhetischer Aspekt). ${ }^{22}$

Im Gegensatz zu üblicher, alltäglicher, meist unbewusst verwendeter Metapher sind bei neuartiger, nicht üblicher metaphorischer (vornehmlich geschriebener) Rede Überraschungsmoment und Heurika-Erleben im Entdecken und Dekodieren des Gemeinten nicht ausgeschlossen. So denkt ein Freund unter Freunden über die eigentliche Bedeutung der (metaphorischen) Aufforderung „schieß los" nicht weiter nach, wenn damit im Kontext des Geschehens lediglich

20 Kritik der Urteilskraft $\S 59$; Ulrich H. J. Körtner, Gottes Wort in Person: Rezeptionsästhetische und metapherntheoretische Zugänge zur Bibel (NeukirchenVluyn: Neukirchener, 2011), 148; Verweis auf Kant auch von Blumenberg, Paradigmen, 15.

21 Mende, „Vorwort“, 9-10. Der vorliegende Aufsatz versteht Gott nicht, aber auch Welt, Mensch, Freiheit und so weiter nicht allein als absolute Metapher, da es etwa durch das Alte und Neue Testament sehr wohl ein „referentielles Wissen“ (Mende, „Vorwort“, 10) von Gott gibt. Durch Selbst-Wahrnehmbarmachung schafft Gott den referentiellen Rückbezug auf sein Wesen und erweist sich als der Entborgene gerade nicht als absolute Metapher.

22 Vergleiche Helge Skirl und Monika Schwarz-Friesel, Metapher (Kurze Einführungen in die Germanistische Linguistik 4; Zweite, aktualisierte Auflage; Heidelberg: Universitätsverlag Winter, 2013), 1-6, dort mit Beispielen; auch Müller, Metapher, 13-14. 
„fang an zu erzählen“ gemeint ist. Ähnliches gilt für den Zwischenruf „dem kannst du nicht das Wasser reichen“, wenn damit wie üblich gesagt werden will, dass eine betreffende Person in betreffender Angelegenheit ein nicht vergleichbar anderes und höheres Niveau einnehme als der Angesprochene. Aber die Äußerung ,in Wien wohnt die Gemütlichkeit“ wird derselbe Zuhörer mit Interesse verfolgen, wenn er noch nie in Wien war, jetzt aber dorthin will und bereits einiges über die Stadt weiß. Die Anmerkung von der in Wien wohnenden Gemütlichkeit könnte in ihm in einem Augenblick das Bild, das Kategorienkonzept von der Allgegenwart einer in Wien gelebten, gar gepflegten Kultur der Gemütlichkeit erzeugen - wiederum metaphorisch alliterativ erläutert.

Entsprechend bilden Metaphern im Verstehen von Lakoff, Johnson und weiteren mehr ab als Begriffe - wie auch Haverkamp, eigentlich kein Vertreter metaphorischer Konzeptualisierungstheorien, aktuell einleitend anmerkt: „Metaphern erfassen mehr, als Begriffe von sich aus begreifen““. ${ }^{23}$ Oder: „Metaphorischer Sprachgebrauch aktiviert eine ganze Heerschar von Ideen und Inferenzen, die im ,eigentlichen' Wort nicht stecken“. ${ }^{24}$ Nach Lakoff, Johnson und weiteren sind Metaphern eher Teil von mentalen Konzepten denn von Sprache allein. Darum können Metaphern, besonders solche in der Form „M ist N“- z.B. „Die Wahrheit ist ein scharfes Schwert“ (Conrad Ferdinand Meyer) als konzeptuelle Metaphern verstanden werden.

Demnach liegt metaphorische Konzeptualisierung dann vor, wenn „das weniger scharf Konturierte in Begriffen des schärfer Konturierten“ konzeptualisiert, „das Nichtphysische in Begriffen des Physischen“25 gedacht und in eins zusammengeführt wird. So wird insgesamt ein neues, beide umgreifendes Konzept erzeugt und damit das nicht-physisch Nicht-Fassbare in neuer Wirklichkeitserschließung fassbar. Metaphorische Konzepte stellen also nicht allein - wie von Lakoff und Johnson auch nur vorläufig vereinfachend zusammengefasst - „Möglichkeiten“ dar, „eine Erfahrung partiell in Begriffen einer anderen Erfahrung zu strukturieren" ${ }^{\prime 26}$, sondern beide Ebenen erzeugen in eins gefasst eine neue Weltwahrnehmung.

In verdichtender Interpetation von Lakoffs und Johnsons Theorie betont Wehling allerdings die notwendige Unterscheidung von Sprache und Denken, ja von der (unbewussten) sprachlichen Anwendung von konzeptullen Metaphern

23 Haverkamp, Metapher, 1. Das Wortspiel geht auf Blumenberg, Paradigmen, 173, vergleiche 188-189, zurück.

24 Wehling, Framing, 72.

25 Lakoff und Johnson, Leben, 73.

26 Ibid., 93. 
und deren Wirkung auf das Denken. Denn es sind die allseits präsenten konzeptuellen Metaphern, die „unser alltägliches Denken strukturieren“ ${ }^{\text {“27 und }}$ die sich im sprachlichen Vollzug im Gehirn festschreiben - und schließlich entsprechend auf Wahrnehmung, Haltung und Handlung des betreffenden Individuums wirken. ${ }^{28}$

\section{B „JHWH, MEIN HIRTE“ ALS LEBENSWIRKLICHKEIT \\ $1 \quad$ Psalm 23 in der Forschungsdebatte}

Für den vorliegenden Aufsatz wird die in 74 Psalmen (der BHS) vorspannend

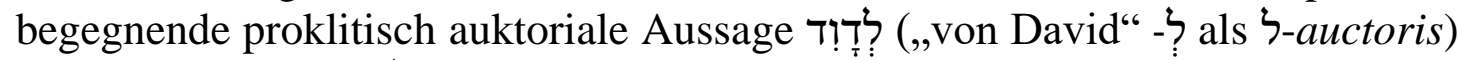
in der Wendung מִזממוֹר לְדְוְד wörtlich aufgenommen, ohne damit festgelegt zu haben, wer mit David als Autor des Psalms, als Psalmist, gemeint ist. ${ }^{29}$ In diesem Sinne werden beide Begriffe (David und Psalmist) im Folgenden verwendet. זמר II Piel (,,[auf]spielen, musizieren, [lob]singen, preisen“), spricht von Liedern, „die vornehmlich mit Begleitung von Saiteninstrumenten gesungen werden". ${ }^{30}$

27 Wehling, Framing, 69.

28 Ibid., 69-74, 75-80 pass.

29 Im Gegensatz zu heute galt in älteren Kommentaren die Identifikation von Ps 23 als von (König) David verfasst für naheliegend, so für Franz Delitzsch, Die Psalmen (Gießen: Brunnen, [1894] 2005), 221; aktuell auch für Alan Millard, "Psalm 23: A Psalm for a Covenant King," in Sprache lieben - Gottes Wort verstehen: Beiträge zur biblischen Exegese (Hg. Walter Hilbrands; Gießen: Brunnen, 2011), 99-108.

30 Wilhelm Gesenius, Hebräisches und Aramäisches Handwörterbuch über das Alte Testament (18. Auflage; Berlin: Springer, 2013), 305, 654; auch Chr. Barth, , זמרr", ThWAT II: 603-612. 
Mindestens seit Gunkel ${ }^{31}$ wird Ps 23 formkritisch als Vetrauenspsalm ${ }^{32}$, als Vertrauensgebet ${ }^{33}$, als Vertrauenslied (des Einzelnen) ${ }^{34}$ aufgefasst. In jedem

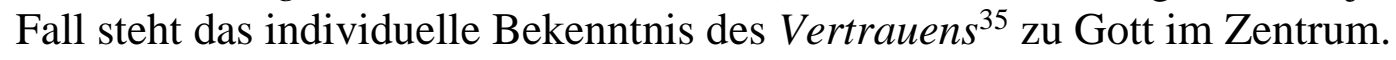

\section{2 „JHWH, mein Hirte“ - Konzeptualisierung und Mapping}

Wehlings beinah bedrohlicher Anklang von der Übermacht unzählbarer sich im Gehirn unbemerkt festgeschriebener konzeptueller Metaphern kann mit Blick auf Ps 23 aber auch eine ermutigend positive, weil vom Psalmisten bewusst gesetzte Richtung anzeigen.

So stellt der Psalmist mit dem (bewusst gewählten) bekannten Wort „JHWH [ist] mein Hirte“ (יהוזה רעִִי:, Ps 23,1) als , ,altorientalische Metapher“36 eine mentale Verknüpfung zwischen dem nicht fassbaren Gott und dem, weil Teil der Lebenswelt des Alten Vorderen Orients seiend, mental fassbaren Hirteund Schafsein, genauer dem Hirtenkönig- und Menschenschaf-Sein her. ${ }^{37}$ Die pronominale Anmerkung ,mein“ unterstreicht, dass David zu allererst sich selbst als Schaf (und Mensch zugleich), als persönliches Eigentum des Hirtenkönigs JHWH erlebt. Das heißt, der Bedeutungsempfänger, der konzeptuelle Zielbereich (auch target domain; conceptual domain (b) usw.) GOTT - ansonsten abstrakt, fern, ungreifbar - wird mit dem Bedeutungsspender, dem konzeptuellen Ursprungsbereich (auch source domain; conceptual domain (a) usw.) MEIN HIRTE für den Leser als persönlich und vertraulich ganz nah und greifbar konzeptualisiert. ${ }^{38}$

31 Hermann Gunkel, Einleitung in die Psalmen: Die Gattungen der religiösen Lyrik Israels (zu Ende geführt von Joachim Begrich; 2. Auflage; Göttingen: Vandenhoeck \& Ruprecht, [1933] 1966).

32 Exemplarisch Hans-Joachim Kraus, Psalmen. (1. Teilband; Neukirchen: Neukirchener, 1960), 188.

33 Exemplarisch Walter Dietrich u.a., Die Entstehung des Alten Testaments (Neuausgabe; Stuttgart: Kohlhammer, 2014), 493.

34 Nina Meyer zum Felde, Hiobs Weg zu seinem persönlichen Gott: Studien zur Interpretation von Psalmentheologie im Hiobbuch (Göttingen: Vandenhoeck \& Ruprecht, 2020), 102-104, 105.

35 Exemplarisch Markus Witte, „Schriften (Ketubim)“, in Grundinformation Altes Testament. Eine Einführung in Literatur, Religion und Geschichte des Alten Testaments (Hg. Jan Christian Gertz; 4. durchgesehene Auflage; Göttingen: Vandenhoeck \& Ruprecht, 2010), 418.

36 Kraus, Psalmen, 189.

37 Um der Einfachheit willen wird im vorliegenden Aufsatz nicht das Bild vom Kleinvieh im Allgemeinen, sondern vom Schaf im Besonderen transportiert.

38 Für die in der Fachliteratur anzutreffende Vielfalt der Begrifflichkeit source domain; conceptual domain (a); Bedeutungsspender; Ursprungsbereich (usw.) 
Allerdings gilt auch für den von David als Bedeutungsempfänger verwendeten Gottesnamen JHWH, dass sich Gott selbst damit Mose (und durch ihn Israel und dem Menschen im Allgemeinen) als treuer und Sünden vergebender Bundesgott begreifbarer, ja konzeptuell erfahrbar gemacht hat. (Exod 3,14; 6,2.6-8; 24,9-13.15-18; 34,6-7) So ist innerhalb des JHWH IST MEIN HIRTE für David JHWH als Bedeutungsempfänger desgleichen nicht mehr ganz abstrakt, fern, ungreifbar. Aber mit dem Bedeutungsspender MEIN HIRTE macht er ihn nochmals in einem entscheidenden Schritt des sein gesamtes Leben durchdringenden persönlichen Naheseins konzeptuell greifbarer, ja erfahrbarer. - Dieser Vorgang metaphorisch übertragender Korrespondenz zwischen Bedeutungsspender und Bedeutungsempfänger wird als mapping bezeichnet.

Der ansonsten unbegreifliche GOTT, der sich selbst als JHWH begreifbarer gemacht hat und von David hier so identifiziert wird, begegnet nun als der seine Schafe (und Menschen), ja als der sein persönliches Menschenschaf David VERSORGENDE, LEITENDE, SCHÜTZENDE. Damit hat sich Gott selbst und hat David GOTT der unnahbaren Aura der absoluten Metapher enthoben. Davids metaphorisches (und doch real gemeintes, weil ganzheitlich erfahrenes) mapping Gottes ist das sein-persönlicher-Hirte-Sein und damit indirekt JHWHs-persönliches-Menschenschaf-Sein. Oder anders: Mit der metaphorischen Zugrundelegung und dem weiteren Verlauf des Psalms malt David dem Hörer und Leser das (reale) Konzept des persönlichen Gottes vor Augen, der nicht allein (königlicher) Hirte seines Volkes ist, sondern Davids persönlicher Hüter.

\section{Gott und König als Hirte des Volkes häufig, als Hirte des Einzelnen selten}

Die altvorderorientalische Literatur und so das Alte Testament erweist vielfach, dass der Herrscher als Hirte seines Volkes begegnet, dessen „Schutz- und Führungsfunktion“39 zuweilen mit Hirtenstab abgebildet ist (vgl. Ps 2,9; Offb 2,27). ${ }^{40}$ Damit ist das Bild vom Herrscher als Hirte eigentlich nicht als Metapher zu verstehen, sondern als vom zeitgenössischen (altvorderorientalischen)

einerseits und target domain; conzeptual domain (b); Bedeutungsempfänger; Zielbereich (usw.) andererseits vergleiche zusammenfassend Böckle, Ijob 28, 85.

39 Bernd Janowski und Klaus Scholtissek, „Gottesbilder“, in Handbuch theologischer Grundbegriffe zum Alten und Neuen Testament (Hg. Angelika Berlejung und Christian Frevel; 5. Auflage; Darmstadt: WBG, [2006] 2016), 244.

40 Wie allein der Suchbegriff Hirte in Otto Kaiser, Texte aus der Umwelt des Alten Testaments (TUAT) (3 Bände + Ergänzungslieferung; Gütersloh: Gütersloher Verlagshaus, 1982-2001; TUAT CD-ROM, 2005) erkennen lässt. Im Alten Testament (anklagend) Jer 2,8; 13,20; 23,1-2; Ezech 34,1-10. 
Rezipienten gefasste und erlebte Realität. Denn zahlreiche Seins- und Handlungsaspekte des Hirten werden vom betreffenden Rezipienten im König gesehen und identifiziert. ${ }^{41}$ So kommen etwa die babylonischen Könige Hammurapi und Nebukadnezar in Inschriften mit der geäußerten Bereitschaft zu Wort, ähnlich Ps 23,2 ihre im Reich weit verstreuten Leute fürsorgend in Hülle und Fülle auf Wiesen und Weiden zu führen. Dabei deuten einschlägige Kommentare das Führen auf Wiesen und Weiden als Metapher für Erzeugung und Erhalt der Sicherheit für des Königs Leute. ${ }^{42}$

Dem anknüpfend stellt Millard insgesamt konkrete Seins- und Handlungsaspekte des Hirten (als Gott oder König) gegenüber seinen Leuten als zu versorgende, leitende, schützende Menschenschafe heraus, wie das einerseits in Ps 23, andererseits in ausnehmender Vielfalt in der Literatur des Alten Vorderen Orients abgebildet ist. Dabei steht für Millard ${ }^{43}$ das HirtenkönigLeuteschafe-Verhältnis als beidseitiges Bundesverhältnis zwischen Oberherr (Suzerän) und Vasall im Fokus, worin in vertraglich festgeschriebener Vereinbarung (wie in altvorderorientalischen Texten vielfach bezeugt) der Oberherr Schutz, der Vasall Loyalität verspricht.

Entsprechend bildet die Metapher von Gott als dem Hirten seines Volkes - und damit von Gott als dem Oberherrn seiner Vasallen - im Alten Testament die übliche Konzeptualisierung Gottes als für den Rezipienten selbstverständliche und damit fass- und erlebbare Realität ab. ${ }^{44}$ Auch in der sonstigen altvorderorientalischen (einschließlich der ägyptischen) Literatur begegnet die metaphorische (und als Realität gefasste und erlebte) Identifikation eines Gottes als Hirte der Menschen, der Völker, der Götter, als Hirte von Unterund Oberwelt. ${ }^{45}$

41 Zusammenfassend Millard, "Psalm 23," 108.

42 Hierzu Millard, "Psalm 23," 100-101.

43 Millard, "Psalm 23," 103 und insgesamt.

44 Zum Beispiel Jes 40,11; 49,10; Jer 23,3-4; Ezech 34,11-19; Sach 11,15-17; Ps 74,1; 79,13; 95,7; 100,3; vergleiche auch Jes 53,6.

45 Zum Beispiel im „Gebet Assurbanipals an den Sonnengott Schamasch“, übersetzt von Wolfram von Soden, TUAT II (Hg. Otto Kaiser; Gütersloh: Gütersloher Verlagshaus, 2005), 772: „Schamasch, Hirte der Menschen, fürstlicher Gott““ (Vers 5); im großen akkadischen Schamasch-Hymnus (Erich Ebeling, AOT, 244-247): „Die Völker der Länder allesamt überwachst du, Die Geschöpfe Êas, des Königs und Fürsten, sind allesamt dir anvertraut. Die Lebenshauch begabten Wesen weidest du allzumal, Du bist ihr Hirte, droben und drunten. [...] Hirte der unteren Welt, Hüter der oberen Welt" (Verse 23-26, 33); in Enüma eliš mit Blick auf Marduk (übersetzt von W.G. Lambert, TUAT III, 601): „Möge er den Pfad der Sterne des Himmels beständig machen und wie Schafe alle Götter weiden“ (Tafel VII, Vers 131). 
Umgekehrt begegnet Gott als persönlicher Hirte einer einzelnen Person selten (Gen 48,15; vgl. Ps 119,176; 121) ${ }^{46}$ Zudem begegnen im Hebräischen Namen, deren Subjekt JHWH ist, üblicherweise nicht mit pronominaler

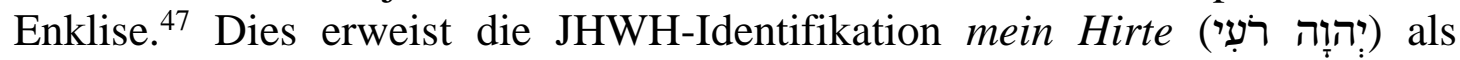
einzigartig. Desgleichen beschreiben die hebräischen Personennamen die Gottheit üblicherweise nicht als Hirte. Wenn in den betreffenden Personennamen das Element רע ( $(r)$ begegnet, dann nicht im Sinne von Hirte (רֶֶ), sondern von Freund (רַע). Wenn allerdings in akkadischen Personennamen das Element $r^{\prime}$ umgekehrt in der Bedeutung von Hirte, aber nicht in der Bedeutung von Freund begegnet, dann erscheint eine solche (interpretierende) Bedeutungsgewichtung für Namen im Hebräischen desgleichen als zumindest möglich. ${ }^{48}$

Aber auch in der sonstigen altvorderorientalischen Literatur begegnet für einige ${ }^{49}$ die persönlich metaphorische Gottesidentifikation mein Hirte kaum etwa aber im altbabylonischen Gedicht „Der Mensch und sein Gott" in Zeile 34 (dort mit "my herdsman" übersetzt). Allerdings merkt Millard ${ }^{50}$ an, dass das

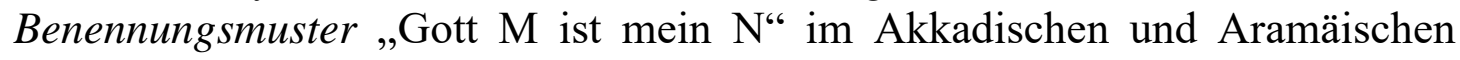
häufiger begegnet („Nabu ist meine Rache“; „Attar ist meine Stütze“) und so desgleichen das Muster „Gott M ist mein Hirte“ (etwa mit Blick auf Schamasch, $\mathrm{Nabu}$ und Zababa im Mittel-Babylonischen, Neu-Assyrischen und NeuBabylonischen). - Hingegen ist offenbar die Wendung mein Gott in der altvorderorientalischen Literatur als stereotype Gottesidentifikation zu bewerten. $^{51}$

\section{JHWH als persönlicher Hirte Davids konzeptualisiert - und heute erfahrbar}

Als der eben dargelegte - und selten konzeptualisierte - persönliche Hirte weidet, leitet, nährt JHWH David als sein Menschenschaf innerlich wie äußerlich liebevoll, überreich und in „Gerechtigkeit“ selbst in tiefster Not und feindlicher Bedrohung. Dabei scheint aus Sicht des vorliegenden Aufsatzes zwar das angedeutete Oberherr-Vasall-Verhältnis eine grundlegende Rolle zu spielen,

46 Vergleiche Kraus, Psalmen, 189; Pierre J. P. van Hecke, "Shepherds and Linguists: A Cognitive-Linguistic Approach to the Metaphor 'God Is Shepherd' in Gen 48,15 and Context," in Studies in the Book of Genesis: Literature, Redaction and History (Hg. André Wénin; Leuven: Peeters, 2001), 479-495.

47 Millard, "Psalm 23," 99. Millard versteht die Formulierung יהוָה רצִי: (,der Herr [ist] mein Hirte") als Benennungsmuster (name pattern).

48 Hierzu mit Belegen Millard, "Psalm 23," 99-100.

49 So für Meyer zum Felde, Hiobs Weg, 19 mit Vorländer.

50 Millard, "Psalm 23," 99.

51 Meyer zum Felde, Hiobs Weg, 19 mit Vorländer. 
dem aber doch das einzigartig persönliche Hirtenkönig-MenschenschafVerhältnis vorgeordnet ist. So will zweiteres im Fokus behalten, ersteres aber nicht aus den Augen gelassen sein.

Dabei spricht Ps 23,5 offenbar mehr schon von Gott als Wirt denn als Hirt. ${ }^{52}$ Aber auch Gott als Wirt scheint für David einen Aspekt Gottes als Hirt widerzuspiegeln. Denn Vers 5 steht desgleichen in der Ausstrahlwirkung von Vers 1 einschließlich von Vers 2-4.

Und nochmals anders gewendet: Mit dem frame (dem Schema, Skript, Deutungsrahmen) MEIN HIRTE wird im (Gehirn, im Geist des) Rezipienten alles abgespeicherte Weltwissen über das Konzept HIRTE, MEIN HIRTE aktiviert und mit GOTT beziehungsweise JHWH exemplarisch durch die folgenden Verse konkretisierend in Verbindung gebracht. Dieser Prozess wird framing genannt. ${ }^{53}$ Darin wird, über das framing hinausgedacht, JHWH dem Rezipienten damals wie heute als der PERSÖNLICHE HIRTENGOTT nahegebracht.

David ist nicht wie ein Menschenschaf, das Gott wie ein Hirte hütet, sondern er ist Menschenschaf Gottes, der sein Hirte ist. Die Metapher von JHWH als mein Hirte spricht nicht von bloßem Vergleich (ist wie), sondern von Identität, von unmittelbar synästhetisch erfahrener Lebenswirklichkeit (ist). Darum sollte man die „Hirtenmetapher“ von Ps 23 ,nicht vorschnell [...] etwa durch den banalen Satz, Gott sorgt für den Menschen““ auflösen, „,sondern als klare Sachverhaltsaussage verstehen, die die Fürsorge Gottes in ein elementares Lebensbild kleidet". 54

52 Delitzsch, Psalmen, 222; Kraus, Psalmen, 187-188; Bernd Janowski, „Der gute Hirte: Psalm 23 und das biblische Gottesbild“, in Ex oriente Lux. Studien zur Theologie des Alten Testaments (Hg. Angelika Berlejung und Raik Heckl; Leipzig: Evangelische Verlagsanstalt, 2012), 253, 256; Beat Weber, Werkbuch Psalmen III: Theologie und Spiritualität des Psalters und seiner Psalmen (Stuttgart: Kohlhammer, 2010), 234. Für Arthur Weiser, Die Psalmen. Erster Teil: Psalm 1-60 (10., unveränderte Auflage; Göttingen: Vandenhoeck u. Ruprecht, [1950] 1987), 154 spricht Ps 23,3-4 zusätzlich von Gott als David geleitender Wanderer. Dem widerspricht aber mindestens Kraus, Psalmen, 187 mit Verweis auf „dein Stock und dein Stab“ (Ps 23,4d), die für ihn eindeutig das „Schützen, Führen und Weiden“ des Hirten bezeichnen.

53 Der neurolinguistische Ansatz des framings, aktuell vor allem vertreten durch Elisabeth Wehling (exemplarisch Wehling, Framing), einer Schülerin George Lakoffs (der ein Schüler Noam Chomskys ist), wird im vorliegenden Aufsatz nicht vertiefend weiterverfolgt.

54 Janowski und Scholtissek, „Gottesbilder“, 244. 
Die Verknüpfung der beiden Kategorienkonzepte $J H W H$ (als mehr abstraktes „nichtphysisches“ Konzept) und Hirte (als mehr konkretes „physisches“ Konzept) in desgleichen pronominaler Unterstreichung (mein - als unmittelbar erfahrenes Konzept: ich als Menschenschaf meines Hirtenkönigs JHWH) vermag im Rezipienten desgleichen ein neues Bild, eine neue Wahrnehmung Gottes in persönlicher synästhetisch ganzheitlicher Erfahrung seines Wesens zu erzeugen beziehungsweise in Form eines konzeptuellen Überraschungsmoments einen neuen Seins- und Wirklichkeitshorizont zu eröffnen. Was David in unmittelbarer Gotteserfahrung beschreibt, vermag dem Rezipienten desgleichen die konzeptuell erfahrene Lebenswirklichkeit, nämlich dass er Schaf (und doch zugleich Mensch) und Gott sein (königlicher) Hirte ist, bewusst zu machen. Dies gilt im Besonderen für den an Jesus Christus Glaubenden, dem jener als der gute Hirte begegnet (Joh 10,1-30; Heb 13,20).

\section{KONZEPTUALISIERUNG DES „DAVIDS-PERSÖNLICHER-HIRTE- SEINS“ JHWHS}

\section{$1 \quad$ Einleitung}

Davids Konzeptualisierung JHWHs als SEIN HIRTE ist in Gott begründet. Denn nur wenn sich Gott David in der Ganzheit des Lebens synästhetisch (das heißt, in der Gesamtheit der inneren und äußeren Sinne) und konzeptuell (das heißt, als diskret fassbares Ergebnis der Gesamtheit von ästhetischer und noetischer Wahrnehmung) als sein persönlicher königlicher Hirte erfahrbar macht, vermag ihn David als solchen zu konzeptualisieren. Dabei prägt der gesellschaftliche Kontext vom allgemein erfahrbaren Oberherr-Vasall-Verhältnis die metaphorische Begriffsfindung Davids in der Übertragung auf das gesellschaftlich selten konzeptualisierte persönliche HirtenkönigMenschenschaf-Verhältnis.

Und nur aus der eben genannten ganzheitlichen Erfahrung Gottes (als eine Form der embodied cognition) vermag David das metaphorisch anklingende (aber real erfahrene) mapping als übertragende Korrespondenz vom Bedeutungsspender (MEIN HIRTE) zum Bedeutungsempfänger JHWH vorzunehmen. Nur so ist das unmittelbare mapping Gottes in dem Davidspersönlicher-Hirte-Sein (Ps 23,1b $\alpha \beta$ ) und damit das mittelbare mapping Davids in dem JHWHs-persönliches-Menschenschaf-Sein (Ps 23,1bүठ) möglich. Mit dem frame MEIN HIRTE macht David den ihm begreifbar gewordenen Gott desgleichen für den Rezipienten im Allgemeinen und für den in der (israelitisch) gottesdienstlichen Glaubensgemeinschaft Lobsingenden im Besonderen greifbar. Das umfassend vielfältige Weltwissen über das Konzept MEIN HIRTE 
Böckle, “Der Herr ist mein Hirte," OTE 34/1 (2021): 189-217 203

wird mit JHWH beziehungweise GOTT in Verbindung und so als vertraulich fürsorgend leitend schützend persönlicher Gott nahegebracht. ${ }^{55}$

Die einzelnen Aspekte des Bedeutungspenders (des frames) MEIN HIRTE im Prozess der korrespondierenden Beutungsübertragung (des mappings) auf den Bedeutungsempfänger JHWH werden mit dem verbleibenden (beinah gesamten) Psalm (Ps 23,1bүס-6) wie folgt konzeptualisiert.

\section{Psalm 23,1 - JHWH, der bleibende Hirte Davids als Grundlegung}

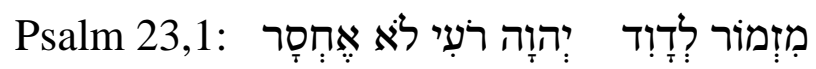

Ein Psalm von David. JHWH ist mein Hirte, nicht leide ich Mangel.

Ein zentraler Aspekt der Konzeptualisierung JHWHs erweist sich bereits in der

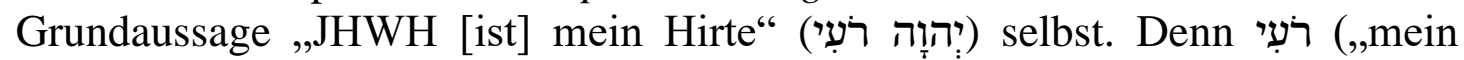
Hirte") bildet ein Partizip (genauer, eine dem Partizip ähnliche Form) von רעה (,weiden, hüten; sorgen, ernähren; [im Partizip:] Hirte") mit pronominaler Enklise der 1. Person Singular ab. Auf Grund des zeitlosen Aspekts des Partizips spricht das persönlicher-Hirte-Sein Gottes für David von einer beständigen, allezeit bestehenden Verfasstheit der göttlichen Vertrautheit und Zugewandtheit. ${ }^{56}$ So formuliert David bereits mit der metaphorisch realen Grundaussage „JHWH [ist] mein Hirte“ die von ihm gleich einer embodied cognition ganzheitlich erfahrene Realität als Seins- und Wirklichkeitshorizont.

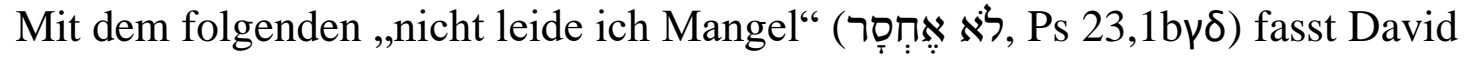
in eins, was das bedeutet, was die Folgen vom persönlicher-Hirte-Sein JHWHs sind - kein Mangel, vielmehr Überfluss, in jeder Lage.

$\mathrm{Zu}$ beachten ist hier desgleichen, dass die Masoreten den Satz ,nicht leide ich Mangel“ (לא אֶחסָר) nicht als eigenes Kolon, sondern als mit ,JHWH [ist] mein Hirte" in Einheit stehenden, vervollständigenden Teil eines Kolons interpretieren. Ersteres spricht davon, wer Gott für David ist, zweiteres davon, was das für David in gesamter erfahrbarer Existenz bedeutet. Und dies - Gottes Hirte-Sein und Davids ohne-Mangel-Sein - gehört in eins zusammen.

55 Entsprechend fokussiert der vorliegende Aufsatz nicht die oben genannten weiteren zentralen Stellen zum Hirtenkönig-Menschenschaf-Verhältnis; desgleichen nicht Beobachtungen zum unmittelbaren Kontext je nach Perspektive einzelner Psalmengruppen, wie die Psalmen 22-26 (Gottesnähe) oder die Psalmen 16-17 und 2223 (individuelle Klage-, Bitt- und Vertrauenspsalmen); auch nicht Verortungen betreffender Psalmen als nachkultisch literarisch in spätnachexilischer Zeit gar im Einfluss durch die Sprüche.

56 Ähnlich Meyer zum Felde, Hiobs Weg, 106. 
Dies führt David mit Ps 23,2-5 im Einzelnen aus. Mit Ps 23,6 formuliert er das Ergebnis familiären Lebens in der beständig vertrauten Gegenwart des königlichen Hirtengottes.

\section{Psalm 23,2-3 - David als Schaf erfährt JHWHs Fürsorge und Leitung als Hirte}

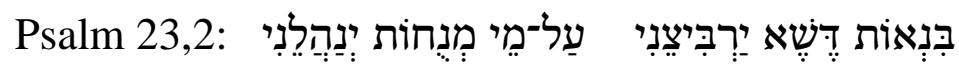

Auf grünen Weidetriften lässt er mich lagern, an Gewässer ruhigster Orte führt er mich.

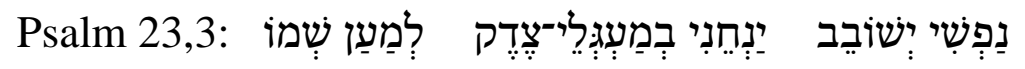

Meine Seele belebt er neu, er leitet mich in Bahnen der Gerechtigkeit um seines Namens willen.

Ps 23,2 evoziert eindeutig das Handeln Gottes als Hirte an David als Schaf. Es bildet Davids ganzheitlich existentielle Erfahrung der fürsorgenden und leitenden Gegenwart Gottes ab. David erlebt real, was metaphorisch anklingt. Die Metapher spiegelt die Realität Gottes in seinem Leben. Gott „lässt“ ihn für

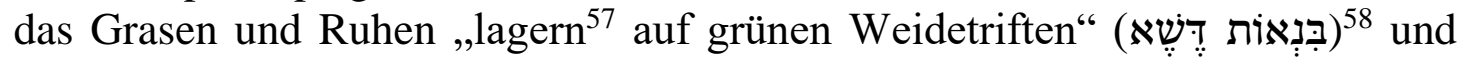

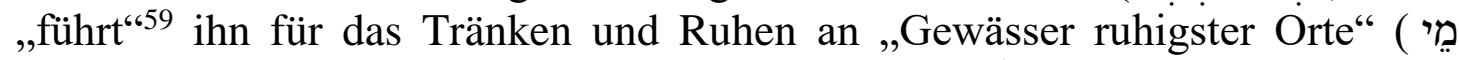
מחנְחוֹת Hifil (Ps 23,3b) bringt נחה Piel (Ps 23,2b) noch mehr das fürsorgende Leiten Gottes als Hirte seines Schafes David zum Ausdruck. Die „ruhigen Wasser“ beziehungsweise „Wasser der Ruhe“ sind desgleichen im Akkadischen bezeugt, auch mit Blick auf einen Beschwörungsspruch, wonach ein Kind so ruhig schlafen möge, wie das Wasser im Wasserbecken ruht. ${ }^{60}$

Der Plural מִנמוּחָה von (,Ruhe[zustand]; Ruheplatz; Wohnsitz“) begegnet im Alten Testament nur noch in Jes 32,18. ${ }^{61}$ In seiner Funktion könnte der Plural auch superlativisch verstanden werden ${ }^{62}$, so dass hiermit die höchste,

57 ירְבְִּיצִנִ (,er lässt mich lagern“, Ps 23,2a) ist Hifil von רבץ (,liegen, lagern, ruhen; lauern [Gen 4,7]“").

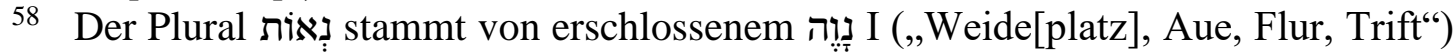
(Gesenius, Handwörterbuch, 791-792).

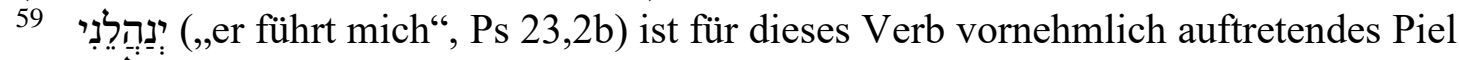
von נהל (,führen, leiten, weiterhelfen, versorgen, ernähren“) (Gesenius, Handwörterbuch, 788; Dr. Frank Matheus, PONS-Kompaktwörterbuch: Althebräisch. Althebräisch - Deutsch (Stuttgart: Ernst Klett Sprachen, 2006), 203.

60 Millard, "Psalm 23," 101.

61 Vergleiche Meyer zum Felde, Hiobs Weg, 108.

62 Vergleiche Delitzsch, Psalmen, 222. 
Böckle, "Der Herr ist mein Hirte," OTE 34/1 (2021): 189-217 205

erfrischendste, ,,anmutigste“ Form von Ruhestätten bezeichnet ist. Damit ist mit der Wendung einmal mehr die besondere, lebenserfüllende und zugleich führende Gottesnähe angezeigt. ${ }^{63}$ Ferner wird auch für den Leser die Gewichtung auf eine Atmosphäre der Ruhe innerlich konzeptuell und unabhängig der Umstände erfahrbar (vgl. Mt 11,28b.29b).

Was metaphorisch gesagt ist und im übertragenen Sinn gelesen werden will (der Psalmist bedarf nicht wörtlich einer Weidetrift und Wasserstelle, denn er ist auch nicht wirklich Schaf), erlebt David doch synästhetisch erfahrbar und ganzheitlich real.

Und eben diese Ebene realen Erlebens und Erfahrens (als Form der embodied cognition) wird mit Ps 23,3 fortgesetzt. Hier scheint eine Unterscheidung Davids von Schaf und Mensch nicht mehr von Relevanz zu sein. Ja, das Gewicht liegt offenbar mehr auf dem Menschen David. ${ }^{64}$ Unabhängig davon scheint aber die Konzeptualisierung Gottes als vertraulich fürsorgend leitender (königlicher) Hirte erhalten zu bleiben.

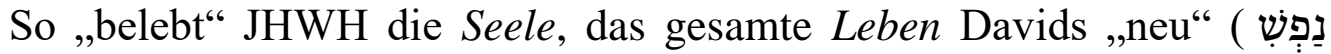

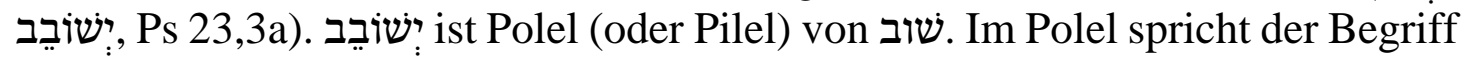
(in positiver Konnotation) von ,[zurück]führen, -bringen; wiederherstellen“. ${ }^{65}$

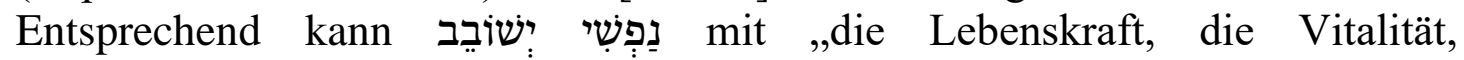
zurückbringen“66, mit „die Seele wieder zu sich selbst, die sich ungleich gewordene wieder in Gleichgewicht bringen, also neu beleben" ${ }^{\text {"67 }}$, interpretiert werden, darum die Übersetzung ,meine Seele belebt er neu“. Die Formulierung setzt voraus, dass sich David davor in gegenteiliger Verfasstheit befand, indem er etwa dem Tod nahestand (vgl. Ps 23,4ab), woraus ihn JHWH gerettet hat. So ist der Hirtengott zugleich ,Lebens- und Rettergott““. ${ }^{68}$

Desgleichen ,leitet“69 JHWH David, ,in Bahnen der Gerechtigkeit“"

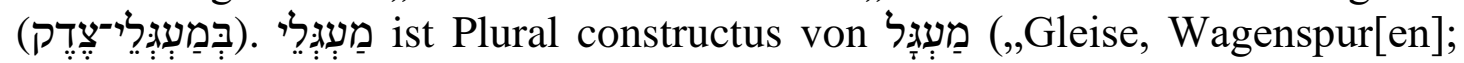
Pfad, Weg“). Die Leitung in Gerechtigkeit ist desgleichen von Assurbanipal, dem hierfür von Schamasch die Menschenschafe gegeben sind, in einer Hymne

63 So Meyer zum Felde, Hiobs Weg, 108 mit Blick auf tempeltheologische Verortungen.

64 Ähnlich Millard, "Psalm 23," 103.

65 Gesenius, Handwörterbuch, 1329; Matheus, Kompaktwörterbuch, 331.

66 Kraus, Psalmen, 189.

67 Delitzsch, Psalmen, 222.

68 Meyer zum Felde, Hiobs Weg, 108-109.

69 נינְחִ (,er leitet mich“, Ps 23,3b) ist Hifil von נחה (,führen, leiten“). Das Wort spricht von Richtung gebendem leiten (Delitzsch, Psalmen, 222). 
bezeugt. $^{70}$ ist hier wohl im Sinne von „Bahn, Wandel, Lebensweise“71 zu

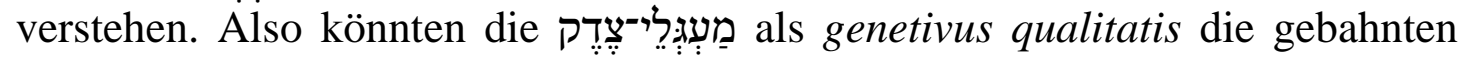
Gerechtigkeitswege Gottes sein, diejenigen Wege des Gerechtseins und gerecht Handelns als Teil der göttlichen Weltordnung ${ }^{72}$, die Gott als Hirtenkönig für das Menschenschaf David gebahnt hat (vgl. Eph 2,10). Das heißt vereinfacht, Gott als Hirte leitet David als Schaf und Mensch in Bedürftigkeit auf guten, rechten, geraden Bahnen und führt ihn zum guten, rechten Ziel. ${ }^{73}$ Dies erweist einmal mehr Gott als den guten, gerechten und damit umfassend fürsorgenden Hirten und König (Joh 10,11.14; vgl. Jes 40,11; Ezech 34,11-16; Heb 13,20; 1 Petr 2,25; 5,4). Denn er leitet David als Schaf und Mensch um seines, um des Hirtengottes

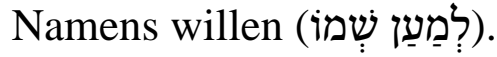

Hier schwingt wohl desgleichen mit, dass Davids Konzeptualisierung Gottes als guter Hirte in der detaillierten Charakterisierung Tendenzen erkennen lässt, die über den Charakter eines (auch guten) natürlichen Hirten und Hirtenkönigs hinausreichen.

\section{Psalm 23,4-5 - David als Menschenschaf erfährt JHWHs Nähe als Hirtenkönig}

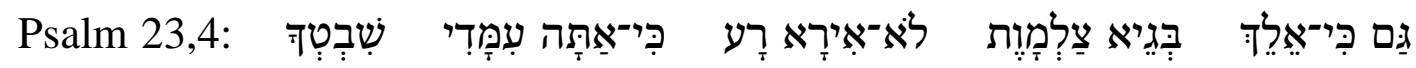

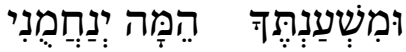

Auch wenn ich wandle im Tal des Todesdunkels, nicht fürchte ich Unheil, denn du bist bei mir, dein Stock und dein Stab, die sind mein Trost.

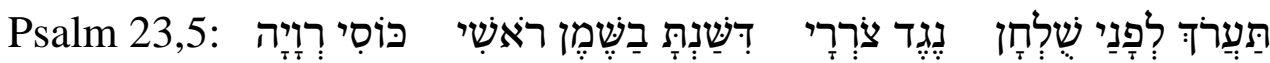

$\mathrm{Du}$ bereitest vor mir einen [gedeckten] Tisch gegenüber den mich Anfeindenden, du salbst mit Öl mein Haupt, mein Becher ist [im] Überfließen.

4a Psalm 23,4 - JHWHs trostreiche Nähe selbst im Lebensdunkel

David wird nun auf zwei Ebenen persönlicher. Einerseits spricht er unmittelbar von sich, von der Möglichkeit des Weges durch Leid und Dunkel nahe dem Tod (Ps 23,4ab), was mit Blick auf Ps 23,3a tatsächlich Teil von Davids Leben sein konnte, ehe ihn Gott daraus wiederbelebend errettete und auf guter Bahn weiterführte (Ps 23,3b). Andererseits spricht David gerade dahingehend Gott als

70 Millard, "Psalm 23," 102.

71 Gesenius, Handwörterbuch, 706.

72 Wovon צֶדָק nach Meinung einiger zumindest auch spricht, so referiert von Klaus Koch, צמדק צsdq", THAT II: 516. Hinweis von Meyer zum Felde, Hiobs Weg, 109.

73 Vergleiche Delitzsch, Psalmen, 222; Janowski, „Hirte,“ 254. 
seinen Hirten unmittelbar in der 2. Person Singular an und bezeugt seine (Gottes) unverbrüchlich treue, trostreiche, dabeiseiende und durch Leid und Todesdunkel mitgehende Anwesenheit (Ps 23,4def). David bezeugt, dass er (David) darin trotz dunkelster Umstände kein Unheil fürchtet (Ps 23,4c), „denn du bist bei

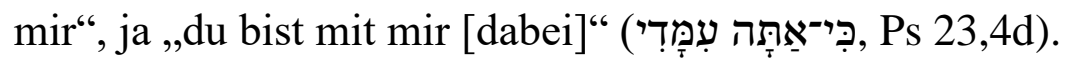

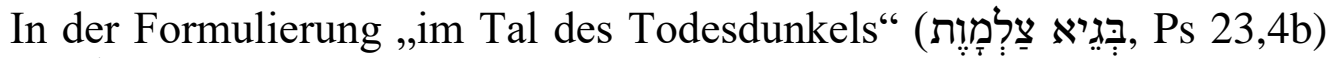

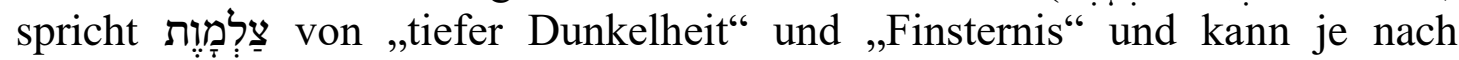
Constructus-Kombination und weiterem Kontext mit dem Eingang ins Totenreich ${ }^{74}$ und mit dem Totenreich selbst ${ }^{75}$ identifiziert werden. Entsprechend

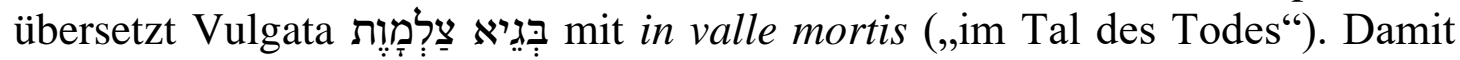
einmal mehr scheint das "Tal der Finsternis“ ein Anspiel auf den Ereignishorizont hin zum Totenreich zu sein, so dass die Interpretation Tal des Todesschattens (oder Todesdunkels), als Grenzlauf des Lebens hin zum Tod und dessen Bedrohlichkeit, nahe liegt. ${ }^{76}$

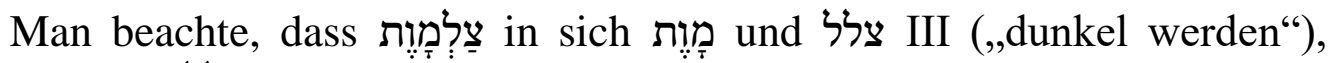
eventuell auch צלל II (,untersinken“), transportiert. Allerdings bildet nach

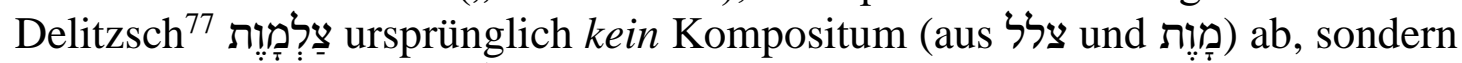
es leitet sich vom Verb צלם ab, das,,sowohl von despotischer Bedrückung als von drückendem lastendem verhüllendem Dunkel“" spricht, wie ja assyrisch șalmu „finster, schwarz" meint.

Im Übrigen fürchten und meiden selbst Könige (wie etwa von Sanherib persönlich bezeugt) mitsamt ihrem Heer neben Wadis auch Täler und Schluchten, weil auf ihnen und durch sie hindurch, auf Grund fernen unbemerkten Regen- oder Schneefalls, unvermittelt ein mächtiger und

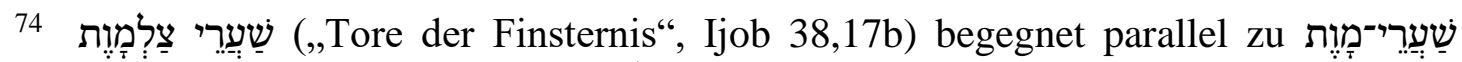
(„Tore des Todes“, Ijob 38,17a), was שַשְערי שְשׁאוֹל (,Tore des Totenreichs“, Jes 38,10; Mt 16,18 Hebrew New Testament) entspricht, weil מָוֶּ 16 mit Mal im Alten Testament (davon hauptsächlich in den Psalmen) für die Bezeichnung des Totenreichs parallel positioniert ist.

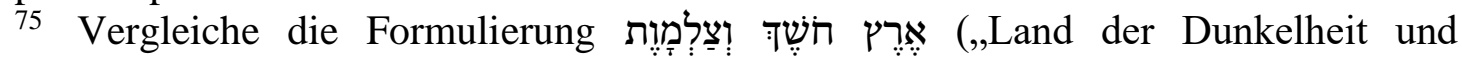

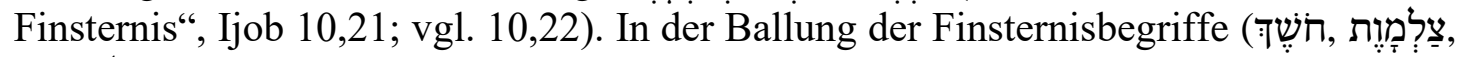
(אפֶּל ,עֵיפָה spricht Hiob vom Totenreich.

$76^{\tau}$ Meyer zum Felde, Hiobs Weg, 110 argumentiert mit Mittmann, dass die Wendung die letzte Bedrohung, die Existenzbedrohung durch den Tod“"symbolisiere. Hierzu und insgesamt zu Ps 23,4 ausführlich Johannes Schnocks, „Metaphern für Leben und Tod in den Psalmen 23 und 88“, in Metaphors in the Psalms (Hg. Pierre J. P. Hecke und Antje Labahn; Leuven: Peeters, 2010), 237-242.

77 Delitzsch, Psalmen, 222. 
vernichtender Wasserschwall heranschießen könnte. ${ }^{78}$ Gott als Hirtenkönig geleitet das Menschenschaf David aber auch auf solch lebensbedrohlichen Wegen und ist immer dabei.

Konkret erweist sich Gottes trostreiche dabeiseiende und mitgehende Gegenwart für das Menschenschaf David in ,dein[em] Stock und dein[em] Stab“

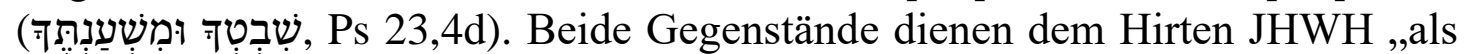
Mittel der Leitung und der Abwehr“. Keule“ sein, ist also „Schutzwaffe“ vor Raubtier und Mensch. מִשְשֶֶֶׁ dient dem Hirten als Stab des Antreibens und Zurückführens zögernder und verirrter Schafe, ist also „Leitstab“. ${ }^{80}$ Entsprechend bilden Stock und Stab metaphorisch Zepterstäbe von mesopotamischen Königen - so von Hammurapi, TukultiNinurta I und anderen - ab, die ihnen als Werkzeuge für Recht, Schutz und (von einem Gott verliehener) Regierungsmacht dienen. ${ }^{81}$

In der unmittelbaren Nähe Gottes als Hirte und hier sichtlich als Hirtenkönig, in der Atmosphäre der ganzheitlich wahrnehmbaren Schutzausübung und Fürsorge Gottes ist David als Menschenschaf getröstet, selbst im Lauf des Lebens an der Grenze zum Tod. Er weiß sich in Sicherheit. ${ }^{82}$ Gottes Nähe, Schutz und Fürsorge ist ihm so innig, so unmittelbar wahrnehmbar gegeben, dass er dies allein mit dem Hirten-Bild zu fassen weiß. Entsprechend könnte (mindestens) Ps 23,4 beide Ebenen der Konzeptualisierung transportieren: Einerseits spricht David zu Gott als den trostreichen Schafhirten mit Schutzwaffe und Leitstab für das ganz praktische, auch durch dunkel bedrohliche Täler verlaufende Leben als Schaf. Andererseits spricht David zu demselben Gott als den trostreichen Hirtenkönig mit den Zepterstäben von Schutzerhalt, Recht und Leitung für das ganz praktische, auch durch mögliches Todesdunkel velaufende Leben als Mensch. Genauer sind die betreffenden Hirten- und Zepterstäbe in Gottes Hand Davids Trost (נחם) ${ }^{83}$, denn sie erweisen die persönliche Gottesfürsorge um David. ${ }^{84}$ So vermag David mit Gott als seinem Hirten (und König) auf Du zu sein. Sein Hirte (und König) ist immer bei ihm. Auch in finsterster Lebenszeit.

78 Vergleiche Millard, "Psalm 23," 102. Die Begründung für der Könige Respekt vor Wadis, Täler und Schluchten nennt Millard nicht.

79 Delitzsch, Psalmen, 222.

80 Vergleiche Kraus, Psalmen, 190.

81 Millard, "Psalm 23," 102-103.

82 Vergleiche Delitzsch, Psalmen, 222.

83 Die apersonale Formulierung, nämlich dass נחם nicht von einem personalen Subjekt abhängt, ist im Alten Testament einzigartig (Meyer zum Felde, Hiobs Weg, 110-111).

84 Hierzu auch Meyer zum Felde, Hiobs Weg, 110-111. 
4b Psalm 23,5 - JHWHs versorgende Nähe selbst in Feindesbedrohung

Mit Ps 23,5 greift David offenbar einmal mehr die real erfahrbare Metapher des altvorderorientalischen Oberherr-Vasall-Bundesverhältnisses auf. Denn einerseits erklärt sich in diesem Kontext der König als Oberherr bereit, seinen Vasallen vor dessen Feinden zu schützen. Es gilt das Prinzip: Deine Feinde sind meine Feinde, aber auch, deine Freunde sind meine Freunde ${ }^{85}$ Und ist dann andererseits der Kampf geschlagen, oder steht er noch bevor, wird des Vasallen Schutz durch den Oberherrn öffentlich, für alle sichtbar, zelebriert. Das signalisiert für den freundlich wie feindlich gesinnten Beobachter, dass der Vasall nicht allein ist, sondern dass er unter der Schirmherrschaft seines Königs steht, wie es vertraglich festgelegt ist. Für David ist - in diesem Bild gedacht der Ort der öffentlich wahrnehmbar gemachten und damit deklarierten Zuwendung Gottes als sein Schirmherr und Hirtenkönig durch den gastfreundschaftlich gedeckten Tisch abgebildet. ${ }^{86}$

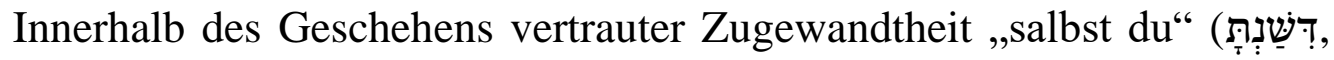
von דשן, Ps 23,5c), so wieder Davids unmittelbar persönliche Anrede Gottes,

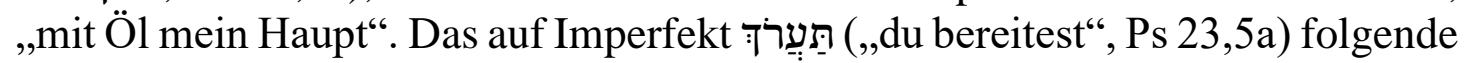

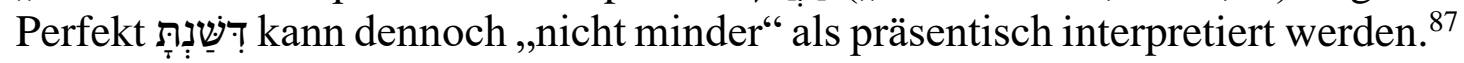
Der Begriff bezeichnet nicht die priesterliche Salbung (wovon gewöhnlich משח spricht), sondern er meint (wie hier in Ps 23 im Piel),,fett, markig machen; laben; für fett halten" (vgl. Ps 45,8), von einer zum Beispiel durch den Gastgeber vorgenommenen Handlung für Wohlbefinden und Freude des Gastes. Dabei wird etwa das Haupt des Gastes mit Öl (das mit besonderen Duftstoffen durchsetzt ist) gesalbt. Wenn es dann herabläuft und -tropft (vgl. Ps 133,2), wird wohl vom Gast selbst, indem er von Hand das Öl verstreicht, das Gesicht (je nachdem einschließlich des Bartes) fett und glänzend gemacht (vgl. Ps 104,15; Lk 7,46; auch Mt 26,6-13 par.). Damit ist desgleichen die trockene Gesichtshaut in klimatisch trockener Umgebung geschmeidig und wohlfühlend geworden. ${ }^{89}$

85 Millard, "Psalm 23," 103-104.

86 Ibid, 104-105, mit Beispielen aus dem Alten Vorderen Orient.

87 Delitzsch, Psalmen, 223.

88 Gesenius, Handwörterbuch, 262; Matheus, Kompaktwörterbuch, 70.

89 Vergleiche auch Darlegung und Abbildung bei Evelina Volkmann, „Salbe, Salbung“, in Calwer Bibellexikon (Hg. Otto Betz, Beate Ego und Werner Grimm; Stuttgart: Calwer Verlag, [2003] 2006); ferner Millard, "Psalm 23," 105; ähnlich Meyer zum Felde, Hiobs Weg, 113-114, allerdings - mit Blick auf den parallelen (nicht in Ps 23 begegnenden) Stamm רוה (Psich laben; sich betrinken“) von רוזיָה (Ps, 23,5d, „Überfluss, [über]voll“) - in der Gewichtung der erquickenden „Festfreude“ durch reichliches Öl im Allgemeinen, nicht des Salbungsgeschehens im Besonderen. 
Aber Gott fügt dem Handeln vertraulicher Zugewandtheit ein zweites

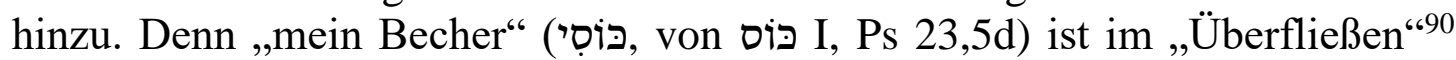
begriffen. Die Wendung „mein Becher“ ist wohl eine Metonymie auf den Inhalt des Bechers, der durch Gottes fürsorgende Präsenz „bis zum Berauschen“ überquillt. ${ }^{91}$ Gott sorgt für David ganzheitlich, in der Gesamtheit der Bedürnnisse, mit Speis und Trank. Und so besteht die Überfülle des Segens Gottes als Hirtenkönig selbst in der Gegenwart der Feinde.

Entsprechend ist wohl der Wechsel der Metapher vom Hirt zum Wirt gar nicht als solcher $\mathrm{zu}$ verstehen. Vielmehr scheint darin einmal mehr die eigentliche Weite der Rede Davids von Gott als seinem persönlichen Hirtenkönig ausgedrückt zu sein. David versteht sich eben nicht allein als des Hirtengottes Schaf, sondern doch als Mensch, den Gott als altvorderorientalischer Hirtenkönig so liebevoll versorgt und bewirtet, dass er sich selbst in der sichtbaren Präsenz der vor ihm gereihten Feinde sicher und geborgen fühlen kann. Und eben das ist umgekehrt für jedermann deklarierend wahrnehmbar gemacht. Davids Feinde sind Gottes Feinde. Und das bezeugt Gott öffentlich in vertraulich fürsorgender Zuwendung seinem Menschenschaf David. Gott tritt nicht als Krieger auf und kämpft nicht vor den Augen Davids gegen dessen Feinde. Vielmehr deckt er ihm - wohl nach tatsächlich geschehenem oder noch bevorstehendem Kampf - in einer dem Psalm eigenen Atmosphäre von Friede und Ruhe, den Tisch, macht für das gemeinschaftliche Mahl Haupt und trockene Gesichtshaut mit Freudenöl (שֶמֶמן שָששוֹ, so Ps 45,8) fett und dient ihm damit in vertraulicher Zugewandtheit.

\section{Psalm 23,6 - JHWHs Segnungen als Verfolger, JHWHs Haus als Wohnung}

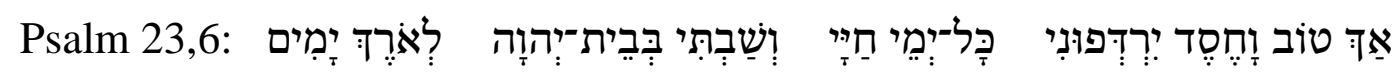

Nur Gutes und Güte werden mir folgen alle Tage meines Lebens; und ich werde wohnen im Haus JHWHs für die Länge der [Lebens-]Tage.

In der metaphorischen, aber real gesellschaftlich anerkannten und persönlich ganzheitlich erfahrenen Konzeptualisierung JHWHs als Hirtenkönig und sich selbst als Menschenschaf, in der gleichzeitigen Aktivierung des kulturellen Kontextes vom Oberherr-Vasall-Bundersverhältnis, formuliert David mit Ps 23,6 eine Art Conclusio. In der treuen Obhut Gottes sind die einzigen und

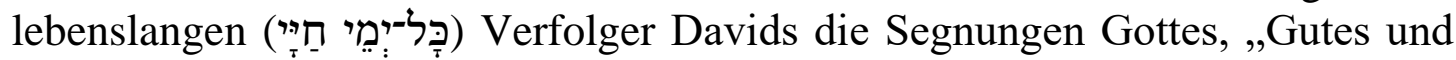

90 Hierzu die vorherige Anmerkung.

91 Delitzsch, Psalmen, 223. 
Güte“ (טוֹ וְחֶסֶ, Ps 23,6a). Testament einzigartig. ${ }^{33}$

Diese Art der Verfolgung schafft entgegen der Bedeutung des Begriffs

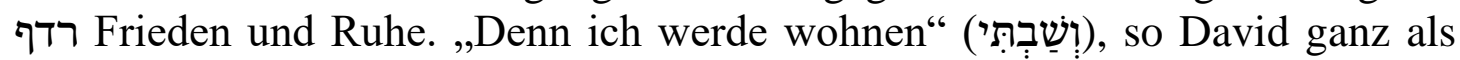

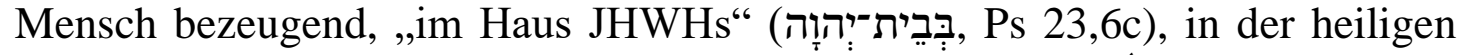
Gegenwart Gottes - und das desgleichen lebenslang (לְארֵּך יְמִים, Ps 23,6d). Nach Delitzsch $^{94}$ muss für die vorgeschlagene Übersetzung nicht erst auf LXX und Symmachus (каi tò катоккعĩv $\mu \varepsilon$, ,und mein Bewohnen [im Haus des Herrn wird

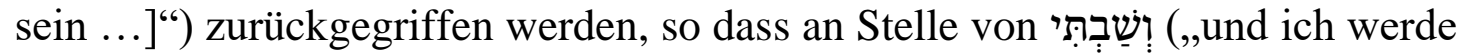

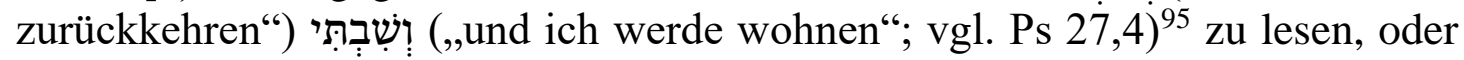

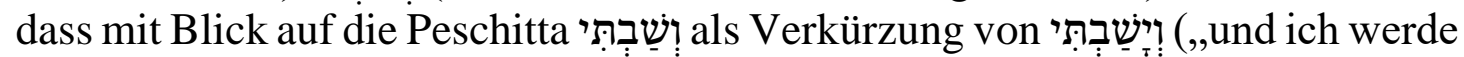
wohnen") zu interpretieren wäre. Denn vielmehr könnte (so Delitzsch weiter)

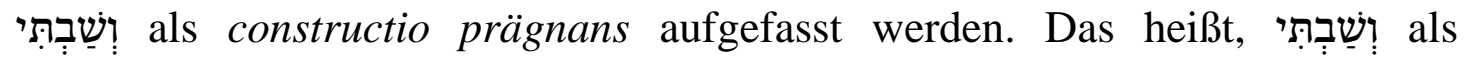
perfectum consecutivum verstanden, spricht davon, dass der ins Haus des Herrn Zurückkehrende als bereits dorthin Zurückgekehrter und damit als dort Wohnender zu interpretieren ist. Und dahingehend ist mit וְשַׁבְתִ (,ich werde

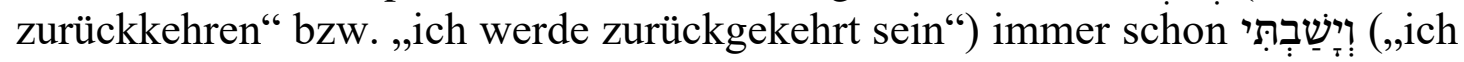
werde wohnen") mitzudenken.

Nicht die Feinde erhalten die Gelegenheit, David zu verfolgen. Denn vor ihnen lässt es ihm sein königlicher Hirtengott JHWH wohl sein. Und damit bleiben die Verfolger Davids auf seinem Lebensweg allein das Gute und Gütige Gottes. Gott als Hirte will David immer nahe sein (Ps 23,4d), würde ihm stets folgen, selbst wenn David ihn flöhe (vgl. Ps 139,8-10), wie der Hirte dem entflohenen Schaf suchend folgt.

Und eben darin besteht Davids Leben in dem beständigen Wohnen in Gottes heiliger Gegenwart, selbst bei immer wieder auftretender Not und (Lebens-)Gefahr (Ps 23,2-5). ${ }^{96}$

92 Vergleiche Delitzsch, Psalmen, 223.

93 Wie SESB Konkordanz den Hinweis von Delitzsch, Psalmen, 223 bestätigt. Stuttgarter Elektronische Studienbibel (SESB 3.0. Ursprünglich herausgegeben von Christof Hardmeier, Eep Talstra, zusammen mit Alan Groves u.a. 3., erweiterte Ausgabe; Stuttgart: Deutsche Bibelgesellschaft, 2009).

94 Delitzsch, Psalmen, 223.

95 שִבְבת ist Infinitiv-constructus-nahe Form mit pronominaler Enklise in der 1. Person Singular von ישב (,sich setzen, sitzen; sich niederlassen, wohnen, bleiben).

96 Ähnlich Meyer zum Felde, Hiobs Weg, 114. 


\section{JHWH, der persönliche Hirte Davids - in Gehalt und Gestalt}

Mit Blick auf Gehalt und Gestalt von Ps 23 lässt sich zusammenfassen: David als Schaf (Ps 23,2-4) und Mensch (Ps 23,4-6) des (königlichen) Hirtengottes erfährt ganzheitlich dessen fürsorgend schützende Leitung an köstliche Lagerorte auf guten Lebensbahnen (Ps 23,2-3), aber desgleichen durch dunkelste Lebenstiefen im Trost der Geborgenheit durch Hirten- und Zepterstäbe (Ps 23,4). Als Verfolgter erfährt David desgleichen die öffentlich bewirtende und deklarierende Zuwendung seines königlichen Hirtengottes in einer Atmosphäre von Sicherheit und Überfülle (Ps 23,5). Entsprechend könnten Ps 23,1 als Grundlegung (fundatio) und 23,6 als Schlussfolgerung (conclusio) verstanden werden, was im Großen den Rahmen Ps 23,1 und 6 und den Kern Ps 23,2-5, für sich wiederum gegliedert in Ps 23,2-3(.4) und (4-)5, erkennen lässt. Wie die Hinzufügung in Klammer verdeutlicht, ist Ps 23,4 je nach thematischer und grammatischer Leserichtung (z.B. „du“ in Vers 4 und 5, obwohl Vers 4 thematisch mehr mit Vers 3 verbunden ist) beiden Unterabschnitten zuordenbar und damit als mögliche mehrschichtige Konkatenation (Verknüpfung) des Vorherigen (Ps 23,1-3) mit dem Folgenden (Ps 23,5-6) erkennbar.

Gott ist Davids persönlicher Hirte, darum erfährt er (David) keinerlei Mangel (Ps 23,1). Sein (königlicher) Hirtengott sorgt für den besten Lagerort und Lebenslauf, selbst in todesgrenznaher Lebenszeit (Ps 23,2-4). Und er sorgt für Überfülle in Gastfreundschaft und Geborgenheit selbst bei sichtlich massiver Verfolgung (Ps 23,5). So sind Gottes gute und gütige Segnungen die einzigen Ruhe und Frieden erzeugenden Verfolger Davids und ist damit das Haus des königlichen Hirtengottes der einzige Wohnort Davids als Ort der Geborgenheit in der Gottesgegenwart auf seinem Lebensweg (Ps 23,6).

\section{ERTRAG: „JHWH IST MEIN HIRTE“ - LEBENSWIRKLICHKEIT ALS LEBENSWIRKUNG}

Die Darlegungen zu Ps 23 illustrieren, dass metaphorische Konzeptualisierung die Welt nicht allein deutet und konstituiert (wie das Lakoff und Wehling unterstreichen), auch nicht für die Erfahrbarkeit des nie erfahrenen Realitätsganzen allein strukturiert (wie das Blumenberg der [absoluten] Metapher zuschreibt). Vielmehr illustriert der Psalm, dass metaphorische Konzeptualisierung die Welt, wie sie sein kann und wie sie ist, in neuem Licht erstrahlen und erfahrbar werden lässt - weil sie (die in neuem Licht erstrahlende und erfahrbare Welt) David als der Urheber der Konzeptualisierung in der Begegnung mit dem sich ihm offenbarenden, ihn beständig geleitenden Gott so erfahren hat. 
Und in anderer Gewichtung: Ähnlich der absoluten Metapher erschließt David mit der Wendung „JHWH ist mein Hirte“ eine neue Welt, ermöglicht den einen gesammelten Blick auf die bestehende komplexe Lebenswelt (mit Gott). Dann aber, gewissermaßen in Auflösung der absoluten Metapher, erschließt David selbst mit den folgenden Worten des Psalms, das heißt mit dem (beinah) gesamten Psalm, die Metapher, tritt hinter sie zurück und eröffnet den Blick auf das Einzelne des Wesens Gottes als persönlicher Hirtenkönig Davids und die Folgen für ihn als Menschenschaf. In der korrespondierenden Präsentation des Nichtphysischen in Begriffen des Physischen begegnen beide Momente, JHWH und MEIN HIRTE, in einer neuen Welt- und Gotteserschließung, führen in der überraschenden Eröffnung eines neuen Seins- und Wirklichkeitshorizonts zu erneuerter konzeptueller Gotteswahrnehmung. Dies geschieht in gleichzeitig ganzheitlich synästhetisch konzeptueller Erfahrung (einer embodied cognition) der Folgen von Gottesgegenwart und Gotteswahrnehmung: Das Menschenschaf David darf - auf Grund der stetig vertraulich fürsorgenden und leitenden Gegenwart Gottes in beständiger Atmosphäre von Ruhe und Frieden auch in Leid und Verfolgung - ohne Mangel sein.

JHWH ist nicht allein Hirte der Völker und vor allem Hirte des Volkes Israel in bundesvertraglicher Vereinbarung (etwa zum Ausdruck gebracht durch das Gemeinschaftsmahl vor JHWH auf dem Berg Sinai, Exod 24,11), sondern er ist desgleichen persönlicher Hirte eines Einzelnen, persönlicher Hirte Davids und aus dessen Perspektive mein persönlicher Hirte (und König) und ich sein persönliches Menschenschaf: In seiner Verantwortung (,um seines Namens willen") lagert er mich, belebt er mich, leitet er mich in ruhigster Atmosphäre (Ps 23,2-3). Selbst in dunkelstem Lauf des Lebens bist du nahe, gehst du mit (und gehst voran), tröstest du mich durch die Zeichen deiner Autorität (Hirtenund Königszepterstäbe). Und in Bedrängnis durch den Feind umsorgst und bewirtest du mich mit Überfülle als auch für andere sichtbares Zeichen deiner Autorität und liebend treuen Zugewandtheit (Ps 23,4-5). Darum leide ich keinen

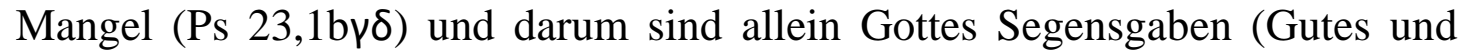
Güte) meine lebenslangen Verfolger zu lebenslangem, friedevollem Wohnen in seiner heiligen Gegenwart (Ps 23,6).

Mit der detaillierten Bezeugung (in zum Teil gleichzeitiger Gottesanrede) der einzelnen Aspekte des Bedeutungsspenders (des frames) MEIN HIRTE im korrespondierenden mapping auf den Bedeutungsempfänger JHWH ermöglicht David für den Rezipienten die ganzheitliche, synästhetisch konzeptuelle Wahrnehmung Gottes, die „Versinnlichung“, das persönliche Miterleben oder Neuerleben dessen, dass Gott auch sein (des Rezipienten) Hirte sein kann. Dies ist möglich, weil sich Gott selbst dem Psalmisten entsprechend wahrnehmbar gemacht und erschlossen hat und sich desgleichen dem Rezipienten wahrnehmbar machen und erschließen kann. 
Und nochmals in eins gefasst: Mit den Worten von Ps 23 wird dem Leser die altvorderorientalische Welt über das vertraulich persönliche Verhältnis zwischen dem Psalmisten und Gott im Bild von Hirtenkönig und Menschenschaf, in Unterlegung des Bundesverhältnisses von Oberherr und Vasall, vor Augen gemalt und als bestehende Möglichkeit der Wirklichkeitswerdung und Anwendung für das persönliche Leben des Lesers inszenierend nahegebracht.

\section{E BIBLIOGRAFIE}

Barth, Chr. „זמר zmr“. Seiten 603-612 in Band II von Theologisches Wörterbuch zum Alten Testament (ThWAT). Herausgegeben von G. Johannes Botterweck und Helmer Ringgren. Stuttgart: Kohlhammer, 1977.

Blumenberg, Hans. Paradigmen zu einer Metaphorologie. Kommentar von Anselm Haverkamp unter Mitarbeit von Dirk Mende und Mariele Nientied. Suhrkamp Studienbibliothek 10. Frankfurt am Main: Suhrkamp, [1960] 2013.

Böckle, Jakob. Ijob 28 in ästhetisch-theologischer Perspektive: Wahrnehmung Gottes und der Weisheit als Herausforderung des Lebens. STB 20. Wien: LIT, 2018.

De Joode, Johan. Metaphorical Landscapes and the Theology of the Book of Job: An Analysis of Job's Spatial Metaphors. VT.S 179. Leiden: Brill, 2018.

Delitzsch, Franz. Die Psalmen. Nachdruck der fünften, überarbeiteten Aufl. von 1894. Gießen: Brunnen, [1894] 2005.

Dietrich, Walter u.a. Die Entstehung des Alten Testaments. ThW 1. Neuausgabe. Stuttgart: W. Kohlhammer, 2014.

Ebeling, Erich. „Babylonisch-assyrische Texte“. Seiten 108-471, in Altorientalische Texte zum Alten Testament (AOT). Herausgegeben von Hugo Gressmann. Zweite, völlig neugestaltete und stark vermehrte Auflage. Berlin: Walter de Gruyter, [1926] 1970. . „Hymnen an den Sonnengott“. Seiten 242-248, in „Babylonisch-assyrische Texte". Herausgegeben von Erich Ebeling.

Gemoll, Wilhelm. Griechisch-deutsches Schul- und Handwörterbuch. Neunte Auflage. Durchgesehen und erweitert von Karl Vretska mit einer Einführung in die Sprachgeschichte von Heinz Kronasser. München: Freytag Verlag [1954] 1988.

Gesenius, Wilhelm. Hebräisches und Aramäisches Handwörterbuch über das Alte Testament. Begonnen von D. Rudolf Meyer unter zeitweiliger, verantwortlicher Mitarbeit von Dr. theol. Udo Rüterwörden und Dr. theol. Johannes Renz, bearbeitet und herausgegeben von Dr. theol. Dr. phil. Herbert Donner, Dr. theol. H.c. 18. Auflage. Berlin: Springer, 2013.

Gunkel, Hermann. Einleitung in die Psalmen: Die Gattungen der religiösen Lyrik Israels. Zu Ende geführt von Joachim Begrich. HK. 2. Auflage. Göttingen: Vandenhoeck \& Rupprecht, [1933] 1966.

Haverkamp, Anselm. Metapher - Mythos - Halbzeug: Metaphorologie nach Blumenberg. Berlin: Walter de Gruyter, 2018.

Haverkamp, Anselm und Dirk Mende. Metaphorologie: Zur Praxis von Theorie. stw 1928. Frankfurt am Main: Suhrkamp, 2009.

Hawley, Lance R. Metaphor Competition in the Book of Job. Journal of Ancient Judaism. Supplements 26. Göttingen: Vandenhoeck \& Ruprecht, 2018. 
Hebrew New Testament. The Trinitarian Bible Society for the Million Testaments Campaigns. London / Philadelphia.

Janowski, Bernd. „Der gute Hirte: Psalm 23 und das biblische Gottesbild“. Seiten 247271, in Ex oriente Lux. Studien zur Theologie des Alten Testaments. Festschrift für Rüdiger Lux zum 65. Geburtstag. Herausgegeben von Angelika Berlejung und Raik Heckl. ABG 39. Leipzig: Evangelische Verlagsanstalt, 2012.

Janowski, Bernd und Klaus Scholtissek. „Gottesbilder“. Seiten 244-246, Handbuch theologischer Grundbegriffe zum Alten und Neuen Testament. Herausgegeben von Angelika Berlejung und Christian Frevel. 5. Auflage. Studienausgabe. Darmstadt: WBG, [2006] 2016.

Jessen, Moiken, Johan Blomberg und Jörg Roche. Kognitive Linguistik. Kompendium DaF / DaZ 2. Tübingen: Narr Francke Attempto, 2018.

Junge, Matthias. Metaphern und Gesellschaft: Die Bedeutung der Orientierung durch Metaphern. Wiesbaden: Verlag für Sozialwissenschaft, 2011.

Kaiser, Otto. Texte aus der Umwelt des Alten Testaments (TUAT + TUAT CD-ROM). 3 Bände + Ergänzungslieferung. Gütersloh: Gütersloher Verlagshaus, 1982$2001+2005$.

Koch, Klaus. צדיק צִdq gemeinschaftstreu / heilvoll sein“. Spalten 507-530, in Band 2 von Theologisches Handwörterbuch zum Alten Testament. Herausgegeben von Ernst Jenni und Claus Westermann. Gütersloh: Chr. Kaiser, [1975] 2004.

Körtner, Ulrich H. J. Gottes Wort in Person: Rezeptionsästhetische und metapherntheoretische Zugänge zur Bibel. Neukirchen-Vluyn: Neukirchener, 2011.

Kövecses, Zoltán. Metaphor: A Practical Introduction. Second Edition. Oxford: University Press, [2002] 2010.

. Extended Conceptual Metaphor Theory. New York: Cambridge University Press, 2020.

Kraus, Hans-Joachim. Psalmen. 1. Teilband. BK XV.1. Neukirchen: Neukirchener, 1960.

Lakoff, George und Mark Johnson. Leben in Metaphern: Konstruktion und Gebrauch von Sprachbildern. Systemische Horizonte. Neunte Auflage. Heidelberg: CarlAuer, [1980, 1997] 2018.

. Philosophy in the Flesh: The embodied mind and its challenge to western thought. New York: Basic Books, 1999.

Lakoff, George und Elisabeth Wehling. Auf leisen Sohlen ins Gehirn: Politische Sprache und ihre heimliche Macht. Systemische Horizonte. Vierte, um ein aktuelles Nachwort ergänzte Auflage. Heidelberg: Carl-Auer, [2008] 2016.

Lambert, W.G. „Enuma Elisch“ (in TUAT III). Seiten 565-602, in TUAT = TUAT CDROM. Herausgegeben von Otto Kaiser. Gütersloh: Gütersloher Verlagshaus, $1982-2001=2005$.

Langenscheidts Großes Schulwörterbuch Lateinisch-Deutsch. Bearbeitet von Dr. Erich Pertsch, auf Grundlage des Menge-Güthling. Erweiterte Neuausgabe. Berlin: Langenscheidt, 1983.

Matheus, Dr. Frank. PONS-Kompaktwörterbuch Althebräisch: Althebräisch-Deutsch. Stuttgart: Ernst Klett Sprachen, 2006.

Mende, Dirk. „Vorwort. Begriffsgeschichte, Metaphorologie, Unbegrifflichkeit“. Seiten 7-32, in Metaphorologie: Zur Praxis von Theorie. Herausgegeben von 
Anselm Haverkamp und Dirk Mende. stw 1928. Frankfurt am Main: Suhrkamp, 2009.

Meyer zum Felde, Nina. Hiobs Weg zu seinem persönlichen Gott: Studien zur Interpretation von Psalmentheologie im Hiobbuch. WMANT 160. Göttingen: Vandenhoeck \& Ruprecht, 2020.

Millard, Alan. "Psalm 23: A Psalm for a Covenant King”. Seiten 99-108, in Sprache lieben-Gottes Wort verstehen: Beiträge zur biblischen Exegese. Festschrift für Heinrich von Siebenthal. Herausgegeben von Walter Hilbrands. BWM 17. Gießen: Brunnen, 2011.

Müller, Horst M. "Embodied Cognition, Embodiment." Seite 174, in Metzler Lexikon Sprache. Herausgegeben von Helmut Glück und Michael Rödel. 5., aktualisierte und überarbeitete Auflage. Stuttgart: J. B. Metzler, 2016.

Müller, Ralph. Die Metapher: Kognition, Korpusstilistik und Kreativität. Poetogenesis. Studien und Texte zur empirischen Anthropologie der Literatur 7. Paderborn: mentis, 2012.

Rickheit, Gert, Sabine Weiss und Hans-Jürgen Eikmeyer. Kognitive Linguistik: Theorien, Modelle, Methoden. UTB 3408. Tübingen: A. Francke, 2010.

Rolf, Eckard. Metaphertheorien: Typologie, Darstellung, Bibliographie. Berlin: Walter de Gruyter, 2005.

Schnocks, Johannes. „Metaphern für Leben und Tod in den Psalmen 23 und 88“. Seiten 235-250, in Metaphors in the Psalms. Herausgegeben von Pierre J. P. van Hecke und Antje Labahn. Leuven: Peeters, 2010.

Skirl, Helge und Monika Schwarz-Friesel. Metapher. Kurze Einführungen in die Germanistische Linguistik 4. Zweite, aktualisierte Auflage. Heidelberg: Universitätsverlag Winter, 2013.

Stuttgarter Elektronische Studienbibel. SESB 3.0. Ursprünglich herausgegeben von Christof Hardmeier, Eep Talstra, zusammen mit Alan Groves u.a. 3., erweiterte Ausgabe. Stuttgart: Deutsche Bibelgesellschaft, 2009. - Mittlerweile als Oberfläche bei Logos Bible Software 8.

Van Hecke, Pierre J. P. "Shepherds and Linguists: A Cognitive-Linguistic Approach to the Metaphor 'God Is Shepherd' in Gen 48,15 and Context'. Seiten 479-495, in Studies in the Book of Genesis: Literature, Redaction and History. Herausgegeben von André Wénin. Leuven: Peeters, 2001. . Metaphor in the Hebrew Bible. BEThL 187. Leuven: Peeters, 2005.

Van Hecke, Pierre J. P. und Antje Labahn. Metaphors in the Psalms. BEThL 231. Leuven: Peeters, 2010.

Verde, Danilo und Antje Labahn. Networks of Metaphors in the Hebrew Bible. BEThL 309. Leuven: Peeters, 2020.

Volkmann, Evelina. „Salbe, Salbung“. Seiten 1161-1162, in Calwer Bibellexikon. Herausgegeben von Otto Betz, Beate Ego und Werner Grimm. Stuttgart: Calwer Verlag, [2003] 2006.

Von Soden, Wolfram. „Gebet Assurbanipals an den Sonnengott Schamasch“ (in TUAT II). Seite 772, in TUAT = TUAT CD-ROM. Herausgegeben von Otto Kaiser. Gütersloh: Gütersloher Verlagshaus, 1982-2001 = 2005 .

Wagenknecht, H. „Kognition“. Seite 1085, in Lexikon der Psychologie. Herausgegeben von Wilhelm Arnold, Hans Jürgen Eysenck und Richard Meili. Augsburg: Bechtermünz Verlag, [1971] 1996. 
Weber, Beat. Werkbuch Psalmen III: Theologie und Spiritualität des Psalters und seiner Psalmen. Stuttgart: Kohlhammer, 2010.

Wehling, Elisabeth. Politisches Framing: Wie eine Nation sich ihr Denken einredet und daraus Politik macht. Edition medienpraxis 14. Köln: Herbert von Halem Verlag, 2016.

Weiser, Arthur. Die Psalmen. Erster Teil: Psalm 1-60. ATD 14. 10. unveränderte Auflage. Göttingen: Vandenhoeck u. Ruprecht, [1950] 1987.

Witte, Markus 2010. „Schriften (Ketubim)“. Seiten 413-586, in Grundinformation Altes Testament. Eine Einführung in Literatur, Religion und Geschichte des Alten Testaments. Herausgegeben von Jan Christian Gertz. 4. durchgesehene Auflage. Göttingen: Vandenhoeck \& Ruprecht, 2010.

Zimbardo, Philip G. Psychologie. 6. neu bearbeitete und erweiterte Auflage. Berlin: Springer, 1995.

Jakob Böckle has recently completed his doctoral studies at the University of South Africa (UNISA). He is in contact with the theological faculties of the University of Vienna. He also teaches German, psychology and philosophy at a Viennese secondary school. Email: jakob.b@gmx.net. ORCID: https://orcid.org/0000-0002-2412-6359. 